\title{
REPRÉSENTATION ASYMPTOTIQUE DES FONCTIONS DE MATHIEU ET DES FONCTIONS D'ONDE SPHÉROIDALES
}

\author{
PAR \\ ROBERT SIPS
}

Introduction. Bien qu'ayant fait, depuis les travaux fondamentaux de E. Mathieu, l'objet d'un nombre assez considérable de recherches, les fonctions de Mathieu restent d'un emploi difficile, principalement par suite de l'impossibilité de les représenter analytiquement d'une manière simple et maniable. La même difficulté se présente, d'une manière plus accentuée encore, dans le cas des fonctions d'onde sphéroidales, car celles-ci ont été beaucoup moins étudiées.

On sait que les fonctions de Mathieu et les fonctions sphéroidales doivent leur importance au fait qu'elles se présentent dans l'étude de l'équation des ondes $\Delta^{2} u+\lambda^{2} u=0$, écrite, dans le cas de deux dimensions, en coordonnées elliptiques ordinaires $(\xi, \eta)$, définies par

$$
x=c \operatorname{Ch} \xi \cos \eta, \quad y=c \operatorname{Sh} \xi \sin \eta,
$$

et, dans le cas de trois dimensions, en coordonnées sphéroidales $(\xi, \eta, \phi)$ définies par

$$
x=c \operatorname{Sh} \xi \sin \eta \cos \phi, \quad y=c \operatorname{Sh} \xi \sin \eta \sin \phi, \quad z=c \operatorname{Ch} \xi \cos \eta
$$

dans le cas de l'ellipsoide allongé, et par

$$
x=c \operatorname{Ch} \xi \cos \eta \cos \phi, \quad y=c \operatorname{Ch} \xi \cos \eta \sin \phi, \quad z=c \operatorname{Sh} \xi \sin \eta
$$

dans le cas de l'ellipsoide aplati.

Dans la plupart des applications, et en particulier dans le présent travail, les deux constantes $\lambda$ et $c$ se présentent toujours simultanément sous la forme du produit $\lambda c$.

Mathieu, lui-même, et ses successeurs immédiats, ont indiqué et utilisé des développements procédant suivant les puissances croissantes de $(\lambda c)^{2}$. Ces développements ne sont cependant convergents que pour des valeurs assez faibles de $(\lambda c)^{2}$. Dans le cas général, on doit faire usage de développements en séries trigonométriques ou en séries de fonctions sphériques, dont les coefficients ne suivent aucune loi de formation simple, et dont des tables ont été données par Goldstein, par Ince et par Stratton, Morse, Chu et Hutner. Ces tables ne peuvent cependant évidemment contenir les valeurs des coefficients que pour des valeurs discrètes de $\lambda c$, et l'interpolation entre ces valeurs est

Presented to the Society, October 30,1948; received by the editors June 16,1947, and, in revised form, March 20, 1948. 
difficile. De plus, le calcul même des coefficients exige la détermination préalable des constantes de séparation, ce qui nécessite la résolution d'une équation qu'on obtient sous la forme peu maniable d'un déterminant infini ou d'une fraction continue illimitée.

Divers auteurs, dont Jeffreys, Ince et surtout Goldstein, ont essayé de former des représentations des fonctions de Mathieu utilisables pour les très grandes valeurs de $\lambda c$. Goldstein, en particulier, a indiqué une série asymptotique qui représente, pour $\lambda c$ assez grand, la constante de séparation de l'équation de Mathieu avec une exactitude remarquable. Le même auteur a obtenu une représentation des fonctions de Mathieu elles-mêmes, mais les expressions formées contiennent au dénominateur des facteurs $\cos ^{m} \eta$ et deviennent infinies pour $\eta=\pi / 2$, alors que la fonction elle-même a une valeur finie en ce point. Les expressions de Goldstein ne peuvent donc pas être utilisées pour la représentation des fonctions de Mathieu dans tout l'intervalle $0-2 \pi$.

Dans ce qui suit, nous donnerons, pour les fonctions de Mathieu et pour les fonctions sphéroidales, des développements asymptotiques qui représentent ces fonctions avec une exactitude d'autant plus grande que la constante $\lambda c$ à elle-même une valeur plus élevée. Les séries obtenues, contrairement a ce qui a lieu avec les séries de Goldstein, représentent les fonctions pour toutes les valeurs réelles de la variable. La précision atteinte dépend évidemment de la valeur de $\lambda c$ et augmente rapidement avec la valeur de cette quantité, mais est, d'autre part, d'autant plus faible que l'ordre, c'est à dire le nombre de zéros de la fonction compris entre 0 et $2 \pi$, est plus élevé. Pour les fonctions fondamentales, donc celles n'ayant pas de zéros, l'erreur relative commise en s'arrêtant aux termes en $(\lambda c)^{-2}$ est inférieure à $0,1 \%$ pour $\lambda c \geqq 5$, ce qui est très suffisant pour la plupart des applications. Cette précision peut d'ailleurs être augmentée en tenant compte d'un plus grand nombre de termes de la série asymptotique, mais, outre que le calcul devient rapidement très compliqué, quoi que ne présentant que des difficultés algébriques, les formules obtenues, elles aussi, perdent la simplicité qui les rend précieuses pour les applications.

Nous avons également formé, pour les constantes de séparation, des développements asymptotiques. Dans le cas des fonctions de Mathieu, la série asymptotique obtenue est identique à celle donnée par Goldstein.

Indiquons, pour terminer, que les relations asymptotiques que nous établirons plus loin montrent que, lorsque $\lambda c$ est très grand, les fonctions sphéroidales allongées et aplaties se comportent d'une manière essentiellement différente. En particulier, on constate que, si on appelle respectivement $a$ et $b$ les constantes de séparation correspondantes, pour $\lambda c$ très grand, $a$ diffère très peu de $(2 p+1) \lambda c-\left(2 p^{2}+2 p+3\right) / 4+m^{2}$, tandis que $b$ se rapproche indéfiniment de $-(\lambda c)^{2}+2(m+2 p+1) \lambda c-\left(2 p^{2}+2 p m+2 p+m+1\right)$, où $m$ et $p$ sont des entiers qui seront définis plus loin. 


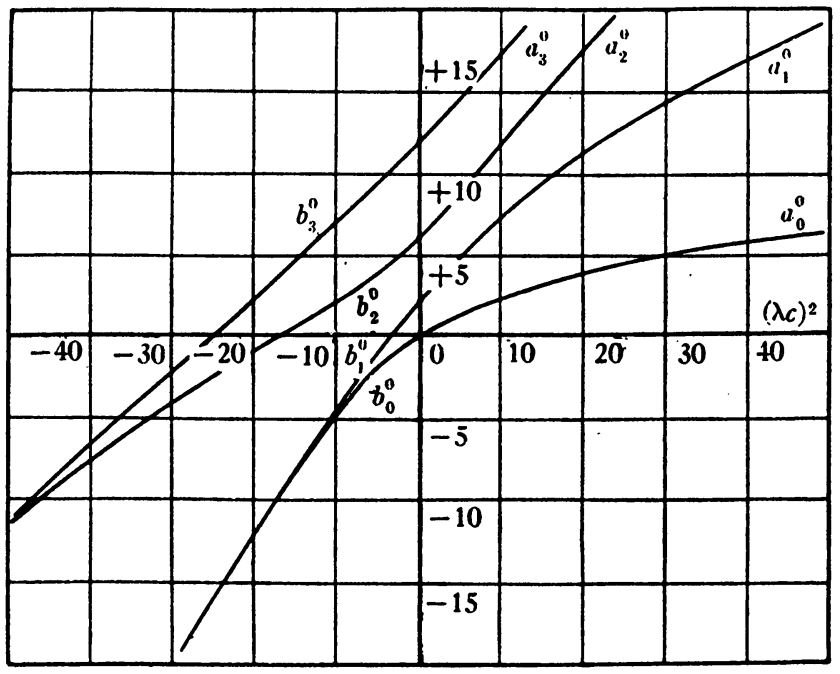

FIG. 1

1. Fonctions de Mathieu. Dans l'équation de Mathieu, écrite sous la forme habituelle

$$
y^{\prime \prime}+\left(a-\frac{(\lambda c)^{2}}{2} \cos 2 \eta\right) y=0
$$

remplaçons la variable indépendante $\eta$ par une nouvelle variable indépendante $\alpha$ définie par

$$
\alpha=(2 \lambda c)^{1 / 2} \cdot \cos \eta .
$$

L'équation (1) prendra alors la forme suivante

$$
y^{\prime \prime}\left(1-\frac{\alpha^{2}}{2 \lambda c}\right)-\frac{\alpha}{2 \lambda c} \cdot y^{\prime}+\left(A-\frac{\alpha^{2}}{4}\right) y=0
$$

où l'on a posé

$$
A=\frac{a+2^{-1}(\lambda c)^{2}}{2 \lambda c}
$$

Introduisons maintenant une série de fonctions $y_{0}(\alpha), y_{1}(\alpha), \cdots, y_{k}(\alpha)$ qui seront définies par les équations différentielles suivantes

$$
y_{0}^{\prime \prime}+\left(n+\frac{1}{2}-\frac{\alpha^{2}}{4}\right) y_{0}=0
$$




$$
\begin{aligned}
y_{1}^{\prime \prime}+\left(n+\frac{1}{2}-\frac{\alpha^{2}}{4}\right) y_{1}= & \left(n+\frac{1}{2}-A\right) y_{0} \\
& +\frac{1}{2 \lambda c}\left(\alpha^{2} y_{0}^{\prime \prime}+\alpha y_{0}^{\prime}\right)-B_{1} y_{0}
\end{aligned}
$$

$$
\begin{aligned}
y_{k}^{\prime \prime}+\left(n+\frac{1}{2}-\frac{\alpha^{2}}{4}\right) y_{k}=( & \left.+\frac{1}{2}-A\right) y_{k-1} \\
& +\frac{1}{2 \lambda c}\left(\alpha^{2} y_{k-1}^{\prime \prime}+\alpha y_{k-1}^{\prime}\right)-B_{k} y_{0}
\end{aligned}
$$

Dans ces équations, $n$ est un nombre entier positif, et $B_{1}, \cdots, B_{k}$ sont des constantes qui seront définies plus loin.

Comme valeur de $y_{0}(\alpha)$, nous prendrons

$$
y_{0}(\alpha)=D_{n}(\alpha)=e^{-\alpha^{2} / 4} H_{n}(\alpha)
$$

où $D_{n}(\alpha)$ est la fonction associée au cylindre parabolique et $H_{n}(\alpha)$ le $n$ ième polynôme d'Hermite. Explicitement

$$
D_{n}(\alpha)=(-1)^{n} e^{\alpha^{2} / 4} d^{n}\left(e^{-\alpha^{2} / 2}\right) / d \alpha^{n} .
$$

D'après les propriétés des fonctions paraboliques, on $a, p$ étant un entier quelconque

$$
\begin{aligned}
& \alpha^{2} D_{p}^{\prime \prime}(\alpha)+\alpha D_{p}^{\prime}(\alpha) \\
& \quad=C_{1, p} D_{p+4}+C_{2, p} D_{p+2}+C_{3, p} D_{p}+C_{4, p} D_{p-2}+C_{5, p} D_{p-4}
\end{aligned}
$$

avec

$$
\begin{aligned}
& C_{1, p}=1 / 4, \quad C_{2, p}=1 / 2, \quad C_{3, p}=\left(2 p^{2}+2 p+1\right) / 4, \\
& C_{4, p}=p(p-1) / 2, \quad C_{5, p}=p(p-1)(p-2)(p-3) / 4 .
\end{aligned}
$$

Bien entendu, dans l'identité (6), les fonctions d'indice négatif doivent être considérées comme identiquement nulles.

Dans ces conditions, le second membre de l'équation $\left(4_{1}\right)$ sera une combinaison linéaire à coefficients constants des fonctions $D_{n+4}, D_{n+2}, D_{n}, D_{n-2}$, $D_{n-4}$. Nous choisirons la valeur de $B_{1}$ de façon à annuler le coefficient de $D_{n}(\alpha)$, et nous prendrons comme valeur de $y_{1}$ une combinaison linéaire à coefficients constants des fonctions $D_{n+4}, D_{n+2}, D_{n-2}, D_{n-4}$. On obtient ainsi

$$
\begin{aligned}
y_{1}(\alpha) & =\frac{1}{2 \lambda c}\left[-\frac{1}{4} C_{1, n} D_{n+4}-\frac{1}{2} C_{2, n} D_{n+2}+\frac{1}{2} C_{4, n} D_{n-2}+\frac{1}{4} C_{5, n} D_{n-4}\right], \\
B_{1} & =n+\frac{1}{2}-A+\frac{C_{3, p}}{2 \lambda c} .
\end{aligned}
$$


Nous introduirons cette valeur de $y_{1}(\alpha)$ dans le second membre de l'équation $\left(4_{2}\right)$, lequel pourra alors être mis sous forme d'une combinaison linéaire à coefficients constants des fonctions $D_{n+8}, \cdots, D_{n-8}$. Nous choisirons, comme plus haut, la valeur de $B_{2}$ de façon à éliminer le terme en $D_{n}(\alpha)$, et nous prendrons comme valeur de $y_{2}$ une combinaison linéaire à coefficients constants des fonctions $D_{n+8}, \cdots, D_{n+2}, D_{n-2}, \cdots, D_{n-8}$. Les fonctions $y_{3}, \cdots, y_{k}$ se calculeront ainsi de proche en proche, en utilisant toujours le même procédé. On vérifiera sans peine que, dans ces conditions, $y_{k}$ sera égal à une combinaison linéaire à coefficients constants des fonctions $D_{n+4 k}, \cdots$, $D_{n+2}, D_{n-2}, D_{n-4 k}$, ne contenant pas de terme en $D_{n}$.

Additionnons maintenant membre à membre les équations $\left(4_{0}\right),\left(4_{1}\right), \cdots$, $\left(4_{k}\right)$, et ajoutons aux deux membres de l'égalité ainsi obtenue l'expression

$$
\left(n+\frac{1}{2}-A\right) y_{k}+\frac{1}{2 \lambda c}\left(\alpha^{2} y_{k}^{\prime \prime}+\alpha y_{k}^{\prime}\right)-B_{k+1} y_{0}
$$

$B_{k+1}$ étant choisi de façon à ce que le coefficient de $D_{n}(\alpha)$ soit nul. En posant

$$
y=y_{0}+y_{1}+\cdots+y_{k}
$$

nous obtiendrons ainsi l'équation différentielle suivante

$$
\begin{aligned}
y^{\prime \prime}+\left(n+\frac{1}{2}-\frac{\alpha^{2}}{4}\right) y+ & \left(n+\frac{1}{2}-A\right) y_{k} \\
& +\frac{1}{2 \lambda c}\left(\alpha^{2} y_{k}^{\prime \prime}+\alpha y_{k}^{\prime}\right)-B_{k+1} y_{0} \\
= & \left(n+\frac{1}{2}-A\right) y+\frac{1}{2 \lambda c}\left(\alpha^{2} y^{\prime \prime}+\alpha y^{\prime}\right) \\
& -\left(B_{1}+B_{2}+\cdots+B_{k+1}\right) D_{n}(\alpha) .
\end{aligned}
$$

L'équation (9) peut encore s'écrire

$$
\begin{aligned}
\left(1-\frac{\alpha^{2}}{2 \lambda c}\right) y^{\prime \prime}-\frac{\alpha}{2 \lambda c} y^{\prime}+\left(A-\frac{\alpha^{2}}{4}\right) y \\
=-\left[\left(n+\frac{1}{2}-A\right) y_{k}+\frac{1}{2 \lambda c}\left(\alpha^{2} y_{k}^{\prime \prime}+\alpha y_{k}^{\prime}\right)-B_{k+1} D_{n}(\alpha)\right] \\
-\left(B_{1}+\cdots+B_{k+1}\right) D_{n}(\alpha) .
\end{aligned}
$$

Nous choisirons maintenant la valeur de $A$, qui est restée jusqu'ici indéterminée, de façon à annuler la somme

$$
B_{1}+B_{2}+\cdots+B_{k}+B_{k+1}
$$

et il nous restera simplement 


$$
\begin{aligned}
(1- & \left.\frac{\alpha^{2}}{2 \lambda c}\right) y^{\prime \prime}-\frac{\alpha}{2 \lambda c}+\left(A-\frac{\alpha^{2}}{4}\right) y \\
& =-\left[\left(n+\frac{1}{2}-A\right) y_{k}+\frac{1}{2 \lambda c}\left(\alpha^{2} y_{k}^{\prime \prime}+\alpha y_{k}^{\prime}\right)-B_{k+1} D_{n}(\alpha)\right] .
\end{aligned}
$$

La somme $B_{1}+\cdots+B_{k+1}$ a la forme suivante

$$
n+\frac{1}{2}-A+\frac{C_{3, n}}{2 \lambda c}+P
$$

où $P$ est un polynôme de degré $k+1$ en $1 / \lambda c$ et $(n+1 / 2-A)$, ne contenant pas de termes du premier degré.

La valeur de $A$ qui nous intéresse est évidemment celle qui se réduit à $n+1 / 2$ pour $1 / \lambda c=0$. Cette valeur de $A$ pourra être représentée par une série de puissances de $(\lambda c)^{-1}$, convergente pour $\lambda c$ assez grand, de la forme suivante

$$
A=n+\frac{1}{2}+\frac{C_{3, n}}{2 \lambda c}+\frac{\gamma_{1}}{(2 \lambda c)^{2}}+\frac{\gamma_{2}}{(2 \lambda c)^{3}}+\cdots
$$

où les $\gamma$ sont des constantes, et ceci peut encore s'écrire

$$
a=-\frac{1}{2}(\lambda c)^{2}+(2 n+1) \lambda c+C_{3, n}+\frac{\gamma_{1}}{2 \lambda c}+\cdots,
$$

$A$ étant une fonction analytique de $(\lambda c)^{-1}$, si l'on arrête le développement en série au terme en $(\lambda c)^{-p}$, le reste sera de la forme $O\left((\lambda c)^{-p-1}\right)$.

Revenons maintenant à l'équation (11). Dans le second membre, remplaçons partout $n+1 / 2-A$ par son développement en série. On voit alors immédiatement que ce second membre sera une combinaison linéaire des fonctions $D_{n+4 k+4}$, $\cdots, D_{n+2}, D_{n-2}, \cdots, D_{n-4 k-4}$ dont les coefficients seront tous $O\left((\lambda c)^{-k-1}\right)$. Or les fonctions $D_{p}(\alpha)$ du cylindre parabolique restent finies pour toutes les valeurs réelles de la variable $\alpha$. Le second membre de (11) sera donc lui-aussi de la forme $O\left((\lambda c)^{-k-1}\right)$, pour $n$ et $k$ donnés, et $\alpha$ ayant une valeur réelle quelconque.

$\mathrm{Si}$, enfin, nous reprenons pour variable indépendante $\eta$, on voit que $y\left((2 \lambda c)^{1 / 2} \cos \eta\right)$ sera une fonction périodique bornée et continue de $\eta$ qui vérifiera l'équation

$$
\frac{d^{2} y}{d \eta^{2}}+\left[a-\frac{(\lambda c)^{2}}{2} \cos 2 \eta\right] y=O\left((\lambda c)^{-k}\right)
$$

Le second membre de cette équation est évidemment une fonction périodique bornée paire et continue de $\eta$.

Introduisons maintenant une nouvelle fonction $Y(\eta)$ qui sera définie par

$$
Y(\eta)=C y\left((2 \lambda c)^{1 / 2} \cos \eta\right)
$$


où $C$ est une constante telle que

$$
\int_{-\pi}^{+\pi} Y^{2}(\eta) d \eta=C^{2} \int_{-\pi}^{+\pi} y^{2}\left((2 \lambda c)^{1 / 2} \cos \eta\right) d \eta= \begin{cases}2 \pi & \text { pour } n=0 \\ \pi & \text { pour } n>0\end{cases}
$$

La fonction $Y(\eta)$ ainsi définie vérifiera à son tour une équation différentielle de la forme suivante

$$
Y^{\prime \prime}+\left(a-\frac{(\lambda c)^{2}}{2} \cos 2 \eta\right) Y=\phi(\eta)
$$

avec

$$
\phi(\eta)=\left(O(\lambda c)^{-k}\right)
$$

On sait, d'autre part, que l'équation de Mathieu (1) possède, pour certaines valeurs bien définies de la constante $a$, des solutions périodiques, de période $2 \pi$, et paires. On appelle généralement $c e_{n}(\eta)$ celle de ces fonctions qui, pour $\lambda c=0$, se réduit à $\cos n \eta$ et qui, de plus, vérifie la condition de normalisation suivante

$$
\int_{-\pi}^{+\pi} c e_{n}^{2}(\eta) d \eta= \begin{cases}2 \pi & \text { pour } n=0 \\ \pi & \text { pour } n>0\end{cases}
$$

et la constante de séparation correspondante est appelée $a_{n}$.

Toute fonction périodique bornée paire peut être développée en série convergente de fonctions $c e_{n}(\eta)$ chaque fois qu'elle peut l'être en série convergente trigonométrique de cosinus des multiples de $\eta$.

La fonction $Y(\eta)$ étant périodique en $\eta$, bornée, paire, et continue ainsi que sa dérivée, pourra être développée en série uniformément convergente, deux fois dérivable, de fonctions $c e_{n}(\eta)$. On aura donc

$$
Y(\eta)=\sum_{m=0}^{\infty} A_{m} c e_{m}(\eta)
$$

Or, d'après (15)

$$
2 A_{0}^{2}+\sum_{m=1}^{\infty} A_{m}^{2}=1 \quad(=2 \text { pour } n=0)
$$

tandis que, en vertu de (16)

$$
\phi(\eta)=\sum A_{m}\left(a_{m}-a\right) c e_{m}(\eta) .
$$

Par conséquent

$$
\int_{-\pi}^{+\pi} \phi^{2}(\eta) d \eta=2 \pi A_{0}^{2}\left(a_{0}-a\right)^{2}+\pi \sum_{m=1}^{\infty} A_{m}^{2}\left(a_{m}-a\right)^{2}=O\left((\lambda c)^{-2 k}\right) .
$$


Soit maintenant $\left(a_{p}-a\right)^{2}$ la plus petite des quantités $\left(a_{m}-a\right)^{2}$, lesquelles sont évidemment toutes différentes les unes des autres. On aura

$$
2 A_{0}^{2}\left(a_{0}-a\right)^{2}+\sum A_{m}^{2}\left(a_{m}-a\right)^{2}>\left(a_{p}-a\right)^{2}\left(2 A_{0}^{2}+\sum A_{m}^{2}\right)=\left(a_{p}-a\right)^{2}
$$

et par conséquent

$$
a_{p}=a+O\left((\lambda c)^{-k}\right) .
$$

La quantité $a$ représente donc asymptotiquement, pour $\lambda c$ très grand, l'une des constantes de séparation de l'équation de Mathieu. La relation (22) montre de plus que, pour $m \neq p$,

$$
A_{m}=O\left((\lambda c)^{-k}\right)
$$

tandis que, comme

$$
1-A_{p}^{2}=2 A_{0}^{2}+\sum_{m \neq p} A_{m}^{2}
$$

on aura aussi

$$
\left(1-A_{p}^{2}\right)=O\left((\lambda c)^{-2 k}\right) \text {. }
$$

Le coefficient $A_{p}$ pouvant être supposé positif, $1+A_{p}$ sera une quantité finie, et par conséquent

$$
A_{p}=1+O\left((\lambda c)^{-2 k}\right) .
$$

De tout ce qui précède, il résulte enfin que

$$
Y(\eta)=c e_{p}(\eta)+O\left((\lambda c)^{-k}\right)
$$

c'est à dire que la fonction $Y(\eta)$ représente asymptotiquement, pour les valeurs élevées de $\lambda c$, l'une des fonctions de Mathieu paires. Il nous reste à rechercher laquelle.

Remarquons pour cela que, pour $\lambda c$ assez grand, $y(\eta)$ différera d'aussi peu qu'on veut de $C D_{n}\left((2 \lambda c)^{1 / 2} \cdot \cos \eta\right)$. On sait, d'autre part, que la fonction $D_{n}(z)$ possède, pour $z$ réel, $n$ zéros réels inférieurs à $(2 n-2)^{1 / 2}$. Si donc $\lambda c \geqq n$ -1 , la fonction $D_{n}\left((2 \lambda c)^{1 / 2} \cdot \cos \eta\right)$, lorsque $\eta$ varie entre $-\pi$ et $+\pi$, aura $n$ zéros réels, et il en sera de même, pour $\lambda c$ assez grand, de $Y(\eta)$. Ceci montre que pour $\lambda c$ supérieur à une certaine valeur, on aura $p=n$, c'est à dire ênfín

$$
\begin{aligned}
Y(\eta) & =c e_{n}(\eta)+O\left((\lambda c)^{-k}\right), \\
a & =a_{n}+O\left((\lambda c)^{-k}\right) .
\end{aligned}
$$

Nous avons donc ainsi obtenu une représentation asymptotique des fonctions de Mathieu paires.

Remarquons incidemment que les formules (28) et (29) justifient à postériori la convention de normalisation (15). 
Nous allons maintenant rechercher la représentation asymptotique correspondante pour les fonctions de Mathieu impaires. On sait que l'équation (1) n'a de solutions périodiques, de période $2 \pi$, impaires, que pour certaines valeurs de la constante de séparation. Nous appellerons, comme d'habitude, $s e_{n}(\eta)$ celle de ces solutions qui, pour $\lambda c=0$, se réduit à $\sin n \eta$ et qui, de plus, vérifie la condition

$$
\int_{-\pi}^{\pi} \operatorname{sen}_{n}^{2}(\eta) d \eta=\pi
$$

La constante de séparation correspondante sera appelée $b_{n}$.

Toute fonction bornée périodique impaire pourra être développée en série convergente de fonctions $s e_{n}(\eta)$ chaque fois qu'elle pourra l'être en série de sinus.

Considérons maintenant une fonction $z_{1}(\eta)$ qui est égale à $y\left((2 \lambda c)^{1 / 2}\right.$ $\cdot \cos \eta)$, pour $0<\eta<\pi$, et égale à $-y\left((2 \lambda c)^{1 / 2} \cdot \cos \eta\right)$ pour $-\pi<\eta<0$. Cette fonction est évidemment périodique et impaire, mais elle est discontinue pour $\eta=0, \pm \pi, \pm 2 \pi, \cdots$.

Pour la rendre continue, nous lui ajouterons une seconde fonction $z_{2}(\eta)$, qui sera égale à $-y\left((2 \lambda c)^{1 / 2}\right)$, pour $0<\eta<\pi$ et à $y\left((2 \lambda c)^{1 / 2}\right)$ pour $-\pi<\eta<0$. La fonction $z=z_{1}+z_{2}$ sera continue, impaire et périodique, et de plus, aura une dérivée continue, car $d z / d \eta=0$ pour $\eta=0, \pm \pi, \cdots$.

Nous introduirons ensuite une nouvelle fonction $Z(\eta)=C_{1} z(\eta)$, où $C_{1}$ est une constante définie par

$$
C_{1}^{2} \int_{-\pi}^{\pi} z^{2}(\eta) d \eta=\pi
$$

La fonction $Z(\eta)$ vérifiera une équation différentielle de la forme

$$
Z^{\prime \prime}+\left(a-\frac{(\lambda c)^{2}}{2} \cos 2 \eta\right) Z=\phi_{1}(\eta)
$$

où $\phi_{1}(\eta)$ est une fonction périodique bornée impaire qui, pour $\lambda c$ suffisamment grand, est telle que

$$
\phi_{1}(\eta)=O\left((\lambda c)^{-k}\right) .
$$

En effet, comme $y\left((2 \lambda c)^{1 / 2}\right)$ contient un facteur $e^{-\lambda c / 2}$, on voit que $z_{2}(\eta)$ n'introduira dans le second membre que des termes $O\left(e^{-\lambda c / 2}\right)$ qui sont négligeables vis-à-vis de ceux en $O\left((\lambda c)^{-k}\right)$.

La fonction $Z(\eta)$ pourra évidemment être développée en série convergente, deux fois dérivable, de fonctions $s e_{n}(\eta)$

$$
Z(\eta)=\sum_{m=1}^{\infty} B_{m} s e_{m}(\eta)
$$


En raisonnant maintenant exactement comme dans le cas des fonctions paires, nous pourrons montrer que

$$
\begin{aligned}
a & =b_{p}+O\left((\lambda c)^{-k}\right), \\
Z(\eta) & =s e_{p}(\eta)+O\left((\lambda c)^{-k}\right)
\end{aligned}
$$

c'est à dire que $Z(\eta)$ et $a$ représentent respectivement l'une des fonctions de Mathieu impaires et la constante de séparation correspondante.

De plus, comme les termes provenant de $z_{2}(\eta)$ sont asymptotiquement négligeables vis-à-vis de ceux provenant de $z_{1}(\eta)$, on voit que l'on pourra prendre comme valeur de $Z(\eta)$ simplement

$$
Z(\eta)=C z_{1}(\eta)
$$

car, dans ce cas, on a évidemment $C=C_{1}$.

Il nous reste maintenant à rechercher la valeur de l'entier $p$. Remarquons pour cela que $Z(\eta)$ possède, en plus des $2 n$ zéros réels de $Y(\eta)$, également un zéro à l'origine, $\eta=0 . Z(\eta)$ a donc, en tout, dans l'intervalle $(-\pi,+\pi)$, $2 n+1$ zéros réels. Il en résulte que l'on aura $p=n+1$, c'est à dire

$$
\begin{aligned}
Z(\eta) & =s e_{n+1}(\eta)+O\left((\lambda c)^{-k}\right), \\
a & =b_{n+1}+O\left((\lambda c)^{-k}\right) .
\end{aligned}
$$

Nous arrivons donc enfin à cette conclusion que, pour $\eta$ compris entre 0 et $\pi, Y(\eta)$ représente asymptotiquement à la fois la fonction $c e_{n}(\eta)$ et la fonction $s e_{n+1}(\eta)$. De même, la quantité $a$ représente asymptotiquement les deux constantes de séparation $a_{n}$ et $b_{n+1}$.

Les deux fonctions $c e_{n}(\eta)$ et $s e_{n+1}(\eta)$ ont la même représentation asymptotique. Or, pour $\eta=0, c e_{n}(\eta)$ n'est pas nul, alors que $s e_{n+1}(\eta)$ est égal à zéro. Pour que l'approximation soit bonne, il faut donc que $c e_{n}(0)$ soit suffisamment petit. Or $c e_{n}(0)$ contient en facteur $e^{-\lambda c / 2}$, quantité qui décroit rapidement quand $\lambda c$ augmente. Il en résulte donc que, pour que les formules asymptotiques représentent les deux fonctions de Mathieu avec une précision satisfaisante, il faudra que $e^{-\lambda c / 2}$ soit petit.

Nous allons maintenant donner les valeurs explicites des différentes expressions dont il a été question plus haut.

On a d'abord, on supposant comme toujours $\alpha=(2 \lambda c)^{1 / 2} \cdot \cos \eta$, et en utilisant la notation (6)

$$
\begin{aligned}
& y_{0}=D_{n}(\alpha) \\
& y_{1}=\frac{1}{2 \lambda c}\left[-\frac{1}{4} C_{1, n} D_{n+4}-\frac{1}{2} C_{2, n} D_{n+3}+\frac{1}{2} C_{4, n} D_{n-2}+\frac{1}{4} C_{5, n} D_{n-4}\right], \\
& y_{2}=\frac{1}{(2 \lambda c)^{2}}\left[\frac{1}{32} C_{1, n} C_{1, n+4} D_{n+8}+\frac{1}{24}\left(C_{1, n} C_{2, n+4}+2 C_{2, n} C_{1, n+2}\right) D_{n+6}\right.
\end{aligned}
$$




$$
\begin{aligned}
& +\frac{1}{16}\left(C_{1, n} C_{3, n+4}+2 C_{2, n} C_{2, n+2}-C_{1, n} C_{3, n}\right) D_{n+4} \\
& +\frac{1}{8}\left(C_{1, n} C_{4, n+4}+2 C_{2, n} C_{3, n+2}-2 C_{4, n} C_{1, n-2}-2 C_{3, n} C_{2, n}\right) D_{n+2} \\
& +\frac{1}{8}\left(-2 C_{2, n} C_{5, n+2}+2 C_{4, n} C_{3, n-2}+C_{5, n} C_{2, n-4}-2 C_{3, n} C_{4, n}\right) D_{n-2} \\
& +\frac{1}{16}\left(2 C_{4, n} C_{4, n-2}+C_{5, n} C_{3, n-4}-C_{3, n} C_{4, n}\right) D_{n-4} \\
& \left.+\frac{1}{24}\left(2 C_{4, n} C_{5, n-2}+C_{5, n} C_{4, n-4}\right) D_{n-6}+\frac{1}{32} C_{5, n} C_{5, n-4} D_{n-8}\right]
\end{aligned}
$$

Dans ces expressions $n+1 / 2-A$ a été remplacé par sa valeur (12). Pour faciliter l'emploi de la formule (40), nous donnerons ci-dessous un tableau des valeurs des coefficients $C$.

\begin{tabular}{|c|c|l|l|l|}
\hline$C_{1, p}=1 / 4$ & $C_{2, p}=1 / 2$ & $C_{3,0}=-1 / 4$ & $C_{4,0}=0$ & $C_{5,0}=0$ \\
& & $C_{3,1}=-5 / 4$ & $C_{4,1}=0$ & $C_{5,1}=0$ \\
& & $C_{3,2}=-13 / 4$ & $C_{4,2}=-1$ & $C_{5,2}=0$ \\
& & $C_{3,3}=-25 / 4$ & $C_{4,3}=-3$ & $C_{5,3}=0$ \\
& & $C_{3,4}=-41 / 4$ & $C_{4,4}=-6$ & $C_{5,4}=6$ \\
& & $C_{3,5}=-61 / 4$ & $C_{4,5}=-10$ & $C_{5,5}=30$ \\
& & $C_{3,6}=-85 / 4$ & $C_{4,6}=-15$ & $C_{5,6}=90$ \\
\hline
\end{tabular}

Ensuite

$$
\begin{gathered}
\alpha=-\frac{(\lambda c)^{2}}{2}+(2 n+1) \lambda c-\frac{2 n^{2}+2 n+1}{4} \\
-\frac{4 n^{3}+6 n^{2}+6 n+2}{32 \lambda c}-\cdots
\end{gathered}
$$

La valeur asymptotique (41) ainsi obtenue pour $a$ est identique à celle donnée par S. Goldstein, et dont cet auteur a vérifié la remarquable exactitude pour les grandes valeurs de $\lambda c$.

Goldstein a obtenu, en même temps que la formule asymptotique (41) une forme asymptotique de la fonction $y(\alpha)$ qui contient, au dénominateur, des termes en $\cos ^{m} \eta$ et qui n'est utilisable que lorsque $\lambda^{2} c^{2} \cos \eta$ est suffisamment grand. La relation entre cette série asymptotique et les fonctions de Mathieu habituelles n'a été obtenue par Goldstein que par un procédé détourné et difficile à justifier et, en tous cas, sa série est impropre à représenter les fonctions de Mathieu dans tout le domaine de variation réel de la variable $\eta$. Il n'en est évidemment pas de même avec notre représentation. 
Il ne nous reste plus maintenant, pour être complet, qu'à calculer la valeur de la constante de normalisation $C$, laquelle, comme nous l'avons vu, est donnée par la relation (15)

$$
\begin{aligned}
C^{2} \int_{0}^{\pi} y^{2}\left((2 \lambda c)^{1 / 2} \cdot \cos \eta\right) d \eta=\frac{C^{2}}{(2 \lambda c)^{1 / 2}} \int_{0}^{(2 \lambda c)^{1 / 2}} y^{2}(\alpha) \frac{d \alpha}{\left(1-\alpha^{2} / 2 \lambda c\right)^{1 / 2}} & =\frac{\pi}{2} \\
(=\pi, \text { pour } n & =0) .
\end{aligned}
$$

On sait que, pour $\alpha<(2 \lambda c)^{1 / 2}$, on a le développement suivant

$$
\begin{aligned}
\left(1-\frac{\alpha^{2}}{2 \lambda c}\right)^{-1 / 2}=1+ & \frac{1}{2} \cdot \frac{\alpha^{2}}{2 \lambda c}+\frac{1 \cdot 3}{2 \cdot 4}\left(\frac{\alpha^{2}}{2 \lambda c}\right)^{2}+\cdots \\
& +\frac{1 \cdot 3 \cdots(2 k-1)}{2 \cdot 4 \cdots 2 k}\left(\frac{\alpha^{2}}{2 \lambda c}\right)^{k}+R=\Sigma+R
\end{aligned}
$$

en représentant par $\Sigma$ la série arrêtée à son $(k+1)^{e}$ terme. La valeur du reste $R$ est donnée par

$$
R=\frac{1 \cdot 3 \cdots(2 k+1)}{2 \cdot 4 \cdots 2 k}\left(1-\frac{\alpha^{2}}{2 \lambda c}\right)^{-1 / 2} \int_{0}^{\alpha /(2 \lambda c)^{1 / 2}} z^{2 k+1}\left(1-z^{2}\right)^{-1 / 2} d z
$$

d'où l'on déduit que

$$
R<K\left(1-\frac{\alpha^{2}}{2 \lambda c}\right)^{-1 / 2}\left(\frac{\alpha^{2}}{2 \lambda c}\right)^{k+1}
$$

où $K$ est une constante qui ne dépend que de $k$. On aura donc

$$
\int_{0}^{(2 \lambda c)^{1 / 2}} y^{2}(\alpha) \cdot R d \alpha<\frac{K}{(2 \lambda c)^{k+1}} \int_{0}^{(2 \lambda c)^{1 / 2}} y^{2}(\alpha) \frac{\alpha^{2 k+2}}{\left(1-\alpha^{2} / 2 \lambda c\right)^{1 / 2}} d \alpha .
$$

Or, $\alpha^{2 k+2} y^{2}(\alpha)$ étant égal à

$$
\left(\alpha^{k+1} y_{0}(\alpha)+\alpha^{k+1} y_{1}(\alpha)+\cdots\right)^{2}
$$

on aura évidemment

$$
\alpha^{k+1} y(\alpha) \leqq \max \left|\alpha^{k+1} y_{0}(\alpha)\right|+\max \left|\alpha^{k+1} y_{1}(\alpha)\right|+\cdots
$$

et comme chaque $y_{p}$, étant égal au produit d'un polynôme en $\alpha$ par $e^{-\alpha^{2} / 4}$ et par $(\lambda c)^{-k}$, reste toujours inférieur à un certain maximum fini, on voit que $\alpha^{k+1} y(\alpha)$ restera inférieur, quel que soit $\alpha$, à un certain nombre $M$ qui décroît lorsque $\lambda c$ augmente. Donc

$$
\frac{K}{(2 \lambda c)^{k+1}} \int_{0}^{(2 \lambda c)^{1 / 2}} y^{2}(\alpha) \frac{\alpha^{2 k+2}}{\left(1-\alpha^{2} / 2 \lambda c\right)^{1 / 2}} d \alpha<\frac{\pi}{2} \cdot \frac{M^{2}}{(2 \lambda c)^{k+1 / 2}}
$$

c'est à dire que 


$$
\begin{aligned}
& \frac{1}{(2 \lambda c)^{1 / 2}} \int_{0}^{(2 \lambda c)^{1 / 2}} y^{2}(\alpha) \frac{d \alpha}{\left(1-\alpha^{2} / 2 \lambda c\right)^{1 / 2}} \\
&=\frac{1}{(2 \lambda c)^{1 / 2}} \int_{0}^{(2 \lambda c)^{1 / 2}} y^{2}(\alpha)\left[1+\frac{1}{2} \cdot \frac{\alpha^{2}}{2 \lambda c}+\cdots\right. \\
&\left.\quad+\frac{1 \cdot 3 \cdots(2 k+1)}{2 \cdot 4 \cdots(2 k)} \cdot\left(\frac{\alpha^{2}}{2 \lambda c}\right)^{k}\right] d \alpha+O\left((\lambda c)^{-k-1}\right)
\end{aligned}
$$

D'autre part, comme une intégrale de la forme

$$
\int_{0}^{(2 \lambda c)^{1 / 2}} e^{-\alpha^{2} / 2} \alpha^{2 r} d \alpha
$$

où $r$ est un entier positif, peut, pour $(2 \lambda c)^{1 / 2}$ très grand, être représentée asymptotiquement de la manière suivante

$$
\int_{0}^{(2 \lambda c)^{1 / 2}} e^{-\alpha^{2} / 2} \alpha^{2 r} d r=\int_{0}^{\infty} e^{-\alpha^{2} / 2} \alpha^{2 r} d r-e^{-\lambda c}(2 \lambda c)^{r-1 / 2}\left[1+O\left((\lambda c)^{-1}\right)\right]
$$

et, comme le second membre de (45) est égal à la somme d'un nombre fini d'intégrales de cette forme, on voit que, finalement, dans le développement asymptotique de $C$, on pourra écrire

$$
\begin{aligned}
\frac{1}{(2 \lambda c)^{1 / 2}} \int_{0}^{(2 \lambda c)^{1 / 2}} & y^{2}(\alpha) \frac{d \alpha}{\left(1-\alpha^{2} / 2 \lambda c\right)^{1 / 2}} \\
= & \frac{1}{(2 \lambda c)^{1 / 2}} \int_{0}^{\infty} y^{2}(\alpha)\left[1+\cdots+\frac{1 \cdot 3 \cdots(2 k-1)}{2 \cdot 4 \cdots(2 k)} \cdot\left(\frac{\alpha^{2}}{2 \lambda c}\right)^{k}\right] d \alpha \\
& +O\left((\lambda c)^{-k-1}\right) .
\end{aligned}
$$

On en déduit que

$$
\begin{aligned}
\frac{1}{C^{2}}= & \frac{1}{\pi} \cdot \frac{1}{(2 \lambda c)^{1 / 2}} \int_{-\infty}^{+\infty} y^{2}(\alpha)\left[1+\frac{\alpha^{2}}{2 \lambda c}+\frac{3 \alpha^{2}}{8(\lambda c)^{2}}+\cdots\right] d \alpha \\
& +O\left((\lambda c)^{-k-1}\right) .
\end{aligned}
$$

En effectuant les calculs et en tenant compte des relations bien connues d'orthogonalité des fonctions paraboliques, on trouve

$$
\frac{1}{C}=\frac{2^{1 / 2}(n !)^{1 / 2}}{(\pi \lambda c)^{1 / 4}}\left[1+\frac{c_{1}}{2 \lambda c}+\frac{c_{2}}{(2 \lambda c)^{2}}+\cdots\right]^{1 / 2}
$$

avec

$$
c_{1}=\frac{2 n+1}{2}
$$




$$
\begin{aligned}
c_{2}= & \frac{(n+1) \cdots(n+4)+n(n-1) \cdots(n-3)}{256} \\
& -\frac{3[(n+1)(n+2)+n(n-1)]}{16}+\frac{9\left(2 n^{2}+2 n+1\right)}{8} .
\end{aligned}
$$

Pour $n=0$, le coefficient $2^{1 / 2} \cdot(n !)^{1 / 2} /(\pi \lambda c)^{1 / 4}$ doit être remplacé par $(\pi \lambda c)^{-1 / 4}$.

Les formules obtenues ci-dessus représentent les fonctions de Mathieu avec une exactitude remarquable pour les valeurs de $\lambda c$ suffisamment grandes pour que les deux fonctions $c e_{n}(\eta)$ et $s e_{n+1}(\eta)$ aient la même valeur au degré d'approximation utilisé. Nous en donnerons ci-dessous quelques exemples.

On trouve sans peine, pour $n=0,1$ et 2 , les valeurs asymptotiques suivantes pour $y(\alpha)$, avec $k=2$.

$n=0$

$$
y=D_{0}-\frac{1}{2 \lambda c}\left(\frac{D_{4}}{16}+\frac{D_{2}}{4}\right)+\frac{1}{(2 \lambda c)^{2}}\left(\frac{D_{8}}{512}+\frac{D_{6}}{64}-\frac{D_{4}}{8}-\frac{9 D_{2}}{16}\right),
$$

$n=1$

$$
y=D_{1}-\frac{1}{2 \lambda c}\left(\frac{D_{5}}{16}+\frac{D_{3}}{4}\right)+\frac{1}{(2 \lambda c)^{2}}\left(\frac{D_{9}}{512}+\frac{D_{7}}{64}-\frac{3 D_{4}}{16}-\frac{5 D_{2}}{16}\right),
$$

$n=2$

$$
\begin{aligned}
y=D_{2} & -\frac{1}{2 \lambda c}\left(\frac{D_{6}}{16}+\frac{D_{4}}{4}+\frac{D_{0}}{2}\right) \\
+ & \frac{1}{(2 \lambda c)^{2}}\left(\frac{D_{10}}{512}+\frac{D_{8}}{64}-\frac{D_{6}}{4}-\frac{41 D_{4}}{32}-\frac{3 D_{2}}{2}\right) .
\end{aligned}
$$

En utilisant ces valeurs, et en tenant compte de (48) et (49), on trouve ensuite facilement que

$$
\begin{aligned}
& \left.\begin{array}{l}
c e_{0}(\pi / 2) \\
\operatorname{se}_{1}(\pi / 2)
\end{array}\right\} \sim(\pi \lambda c)^{1 / 4} \\
& \cdot\left(1+\frac{1}{32 \lambda c}+\frac{81}{2048(\lambda c)^{2}}\right) /\left(1+\frac{1}{4 \lambda c}+\frac{27}{128(\lambda c)^{2}}\right)^{1 / 2}, \\
& \left.\begin{array}{l}
c e_{2}(\pi / 2) \\
s e_{3}(\pi / 2)
\end{array}\right\} \sim-\frac{1}{2}(\pi \lambda c)^{1 / 4} \\
& \cdot\left(1+\frac{5}{32 \lambda c}+\frac{921}{2048(\lambda c)^{2}}\right) /\left(1+\frac{5}{4 \lambda c}+\frac{429}{128(\lambda c)^{2}}\right)^{1 / 2} .
\end{aligned}
$$

On trouvera dans le tableau ci-dessous les valeurs de ces expressions pour différentes valeurs de $\lambda c$, ainsi que les valeurs exactes correspondantes des 
fonctions de Mathieu obtenues par calcul direct à partir des coefficients des séries trigonométriques donnés par Goldstein. (Rappelons que, dans ses calculs, Goldstein utilise, au lieu de $\lambda c$, un coefficient $q$ dont la valeur est donnée par $(\lambda c)^{2}=32 q$.)

Vérification numérique de la formule (51)

\begin{tabular}{|c|c|c|c|c|c|c|}
\hline$q=$ & 0,5 & 2 & 4 & 10 & 30 & 100 \\
\hline$\lambda c=$ & 4 & 8 & $8\left(2^{1 / 2}\right)$ & $(320)^{1 / 2}$ & $(960)^{1 / 2}$ & $(3200)^{1 / 2}$ \\
\hline$c e_{0}(\pi / 2)$ exact & 1,82713 & 2,21093 & 2,42046 & 2,72322 & 3,13136 & 3,6450 \\
\hline Valeur approchée & 1,83401 & 2,21127 & 2,42048 & 2,72323 & 3,13134 & 3,64503 \\
\hline$s e_{1}(\pi / 2)$ exact & 1,83528 & 2,21095 & 2,42048 & 2,72324 & 3,13135 & 3,64508 \\
\hline$-c e_{2}(\pi$ & 0,68630 & 1,03281 & 1,16 & 1,32942 & 1,54543 & 1,81002 \\
\hline Valeur approchée & 0,77139 & 1,03104 & 1,15688 & 1,32676 & 1,54421 & 1,80955 \\
\hline
\end{tabular}

On constate immédiatement, comme il fallait s'y attendre, que l'erreur commise est d'autant plus faible que $\lambda c$ est plus grand. Pour les valeurs quelque peu élevées de $\lambda c$, l'exactitude est remarquable, étant donnée la simplicité des formules utilisées. D'autre part, plus $n$ devient grand, plus l'erreur commise devient importante, ce qui est tout à fait normal, puisque, comme nous l'avons déja fait remarquer plus haut, la même formule asymptotique représente $c e_{n}(\eta)$ et $s e_{n+1}(\eta)$, et ne peut donc donner une bonne approximation que lorsque $\lambda c$ est assez grand pour que les valeurs de ces deux fonctions ne diffèrent plus sensiblement.

L'examen de la formule asymptotique générale montre, par suite de la présence du facteur $e^{-\alpha^{2} / 4}=e^{-\lambda c \cos ^{2} \eta / 2}$, que lorsqu'on fait croître indéfiniment $\lambda c$ en conservant $n$ constant, les fonctions $c e_{n}(\eta)$ et $s e_{n}(\eta)$ tendent toutes deux uniformément vers zéro pour toutes les valeurs de $\eta$ différentes de $k \pi \pm \pi / 2$.

Pour $\eta=\pi / 2$, les fonctions $c e_{n}(\eta)$ et $s e_{n+1}(\eta)$ possèdent lorsque $n$ est pair, un maximum qui croît indéfiniment avec $\lambda c$, de telle manière que

$$
\lim _{\lambda c \rightarrow \infty} \frac{c e_{n}(\pi / 2)}{(\lambda c)^{1 / 4}}=\lim _{\lambda c \rightarrow \infty} \frac{s e_{n+1}(\pi / 2)}{(\lambda c)^{1 / 4}}=(-1)^{n / 2} \frac{\pi^{1 / 4}(n !)^{1 / 2}}{2^{(n+1) / 2}\left(\frac{n}{2} !\right)} .
$$

Si $n$ est impair, $c e_{n}(\eta)$ et $s e_{n+1}(\eta)$ sont évidemment nuls pour $\eta=\pi / 2$ quel que soit $\lambda c$, mais leurs dérivées en ce point croissent indéfiniment avec $\lambda c$, de telle manière que

$$
\lim _{\lambda c \rightarrow \infty} \frac{c e_{n}^{\prime}(\pi / 2)}{(\lambda c)^{3 / 4}}=\lim _{\lambda c \rightarrow \infty} \frac{s e_{n+1}^{\prime}(\pi / 2)}{(\lambda c)^{3 / 4}}=(-1)^{(n+1) / 2} \frac{\pi^{1 / 4}(n !)^{1 / 2}}{2^{(n-1) / 2}\left(\frac{n-1}{2} !\right)} .
$$


D'autre part, pour $0<\eta<\pi$ les fonctions $c e_{n}(\eta)$ et $s e_{n+1}(\eta)$ possèdent toutes deux $n$ zéros. Si $n$ est impair, $\eta=\pi / 2$ est un de ces zéros, tandis que pour $n$ pair, $c e_{n}^{\prime}(\pi / 2)=s e_{n+1}^{\prime}(\pi / 2)=0$. Lorsque $\lambda c$ croît indéfiniment les zéros (différents de $\eta=0$ et $\pi$ pour $s e_{n}(\eta)$ ) se rapprochent indéfiniment de $\eta=\pi / 2$, ainsi que les maxima et les minima qui les séparent.

Il est à peine nécessaire de faire remarquer que les formules données plus haut pourront également être utilisées pour calculer les valeurs approchées des zéros des fonctions de Mathieu et de leurs dérivées.

2. Fonctions d'onde sphéroidales. A. Ellipsoide allongé. Dans l'équation différentielle des fonctions d'onde sphéroidales relatives à l'ellipsoide de révolution allongé

$$
\frac{1}{\sin \eta} \cdot \frac{d}{d \eta}\left(\sin \eta \cdot \frac{d H}{d \eta}\right)+\left(A+\lambda^{2} c^{2} \sin ^{2} \eta-\frac{m^{2}}{\sin ^{2} \eta}\right) H=0
$$

posons d'abord $\mu=\cos \eta, a=A+(\lambda c)^{2}$, ce qui donnera

$$
\frac{d}{d \mu}\left[\left(1-\mu^{2}\right) \frac{d H}{d \mu}\right]+\left(a-\lambda^{2} c^{2} \mu^{2}-\frac{m^{2}}{1-\mu^{2}}\right) H=0 .
$$

Faisons ensuite

$$
H=\left(1-\mu^{2}\right)^{m / 2} U
$$

l'équation différentielle deviendra alors

$$
\left(1-\mu^{2}\right) \frac{d^{2} U}{d \mu^{2}}-2(m+1) \frac{d U}{d \mu}+\left[a-m(m+1)-\lambda^{2} c^{2} \mu^{2}\right] U=0 .
$$

Changeons enfin encore un fois de variable indépendante en posant

$$
\mu=\alpha /(2 \lambda c)^{1 / 2}
$$

ce qui donne à l'équation la forme finale qui suit

$$
\left(1-\frac{\alpha^{2}}{2 \lambda c}\right) U^{\prime \prime}-\frac{m+1}{\lambda c} \alpha U^{\prime}+\left(K-\frac{\alpha^{2}}{4}\right) U=0
$$

avec

$$
K=\frac{a-m(m+1)}{2 \lambda c}=\frac{A+\lambda^{2} c^{2}-m(m+1)}{2 \lambda c} .
$$

Nous allons maintenant former une suite de fonctions $u_{0}(\alpha), u_{1}(\alpha), \cdots$, $u_{k}(\alpha)$, qui seront définies par les équations différentielles suivantes

$$
u_{0}^{\prime \prime}+\left(p+\frac{1}{2}-\frac{\alpha^{2}}{4}\right) u_{0}=0,
$$




$$
u_{1}^{\prime \prime}+\left(p+\frac{1}{2}-\frac{\alpha^{2}}{4}\right) u_{1}=\left(p+\frac{1}{2}-K\right) u_{0}
$$

$$
+\frac{1}{2 \lambda c}\left[\alpha^{2} u_{0}^{\prime \prime}+2(m+1) \alpha u_{0}^{\prime}\right]-B_{1} u_{0},
$$

$$
\begin{aligned}
u_{k}^{\prime \prime}+\left(p+\frac{1}{2}-\frac{\alpha^{2}}{4}\right) u_{k}=( & \left.+\frac{1}{2}-K\right) u_{k-1} \\
& +\frac{1}{2 \lambda c}\left[\alpha^{2} u_{k-1}^{\prime \prime}+2(m+1) \alpha u_{k-1}^{\prime}\right]-B_{k} u_{0}
\end{aligned}
$$

avec

$$
u_{0}(\alpha)=D_{p}(\alpha)
$$

$p$ étant un entier positif, et les $B$ étant des constantes qui seront définies plus loin.

On vérifie facilement que, si $D_{q}(\alpha)$ est la fonction parabolique d'ordre $q$ ( $q$ entier), on aura l'identité

$$
\begin{aligned}
& \alpha^{2} D_{q}^{\prime \prime}(\alpha)+2(m+1) \alpha D_{q}^{\prime}(\alpha) \\
& \quad=C_{1, q} D_{q+4}+C_{2, q} D_{q+2}+C_{3, q} D_{q}+C_{4, q} D_{q-2}+C_{5, q} D_{q-4}
\end{aligned}
$$

avec

$$
\begin{aligned}
& C_{1, q}=1 / 4, \quad C_{2, q}=-m, \\
& C_{3, q}=-\frac{2 q^{2}+2 q+3}{4}-m, \quad C_{4, q}=m q(q-1), \\
& C_{5, q}=\frac{q(q-1)(q-2)(q-3)}{4}
\end{aligned}
$$

les fonctions d'ordre négatif devant être considérées comme identiquement nulles.

Dans ces conditions, le second membre de $\left(60_{1}\right)$ sera une combinaison linéaire des fonctions $D_{p+4}, D_{p+2}, D_{p}, D_{p-2}, D_{p-4}$ à coefficients constants. Nous choisirons la valeur de $B_{1}$ de façon à annuler le coefficient de $D_{p}(\alpha)$, et nous prendrons comme solution $u_{1}(\alpha)$ une combinaison linéaire des fonctions $D_{p+4}, D_{p+2}, D_{p-2}, D_{p-4}$, ce qui nous donnera

$$
\begin{aligned}
u_{1}(\alpha) & =\frac{1}{2 \lambda c}\left[-\frac{1}{4} C_{1, p} D_{p+4}-\frac{1}{2} C_{2, p} D_{p+2}+\frac{1}{2} C_{4, p} D_{p-2}+\frac{1}{4} C_{5, p} D_{p-4}\right], \\
B_{1} & =p+\frac{1}{2}-K+\frac{C_{3, p}}{2 \lambda c} .
\end{aligned}
$$

Nous introduirons ensuite cette valeur de $u_{1}(\alpha)$ dans $\left(60_{2}\right)$, et nous en 
déduirons, par le même procédé, la valeur de $u_{2}(\alpha)$. Nous calculerons de même $u_{3}(\alpha), \cdots, u_{k}(\alpha)$.

On vérifiera sans peine que, dans ces conditions, $u_{k}(\alpha)$ sera une combinaison linéaire à coefficients constants des fonctions $D_{p+4 k}, D_{p+4 k-2}, \cdots$, $D_{p-4 k}$. Additionnons maintenant membre à membre les équations $\left(60_{0}\right)$, $\left(60_{1}\right), \cdots,\left(60_{k}\right)$ et ajoutons aux deux membres la quantité

$$
\left[p+\frac{1}{2}-K\right] u_{k}+\frac{1}{2 \lambda c}\left[\alpha^{2} u_{k}^{\prime \prime}+2(m+1) \alpha u_{k}^{\prime}\right]-B_{k+1} u_{0}
$$

$B_{k+1}$ étant choisi de façon à annuler le coefficient de $D_{p}(\alpha)$. En posant

$$
u=u_{0}+u_{1}+\cdots+u_{k}
$$

ceci donnera

$$
\begin{aligned}
u^{\prime \prime}+\left(p+\frac{1}{2}-\frac{\alpha^{2}}{4}\right) u= & \left(p+\frac{1}{2}-K\right) u+\frac{1}{2 \lambda c}\left[\alpha^{2} u^{\prime \prime}+2(m+1) \alpha u^{\prime}\right] \\
& +B_{k+1} u_{0}-\left(p+\frac{1}{2}-K\right) u_{k} \\
& -\frac{1}{2 \lambda c}\left[\alpha^{2} u_{k}^{\prime \prime}+2(m+1) \alpha u_{k}^{\prime}\right] \\
& -\left[B_{1}+B_{2}+\cdots+B_{k+1}\right] u_{0} .
\end{aligned}
$$

L'équation précédente peut encore s'écrire

$$
\begin{aligned}
u^{\prime \prime}(1 & \left.-\frac{\alpha^{2}}{2 \lambda c}\right)-\frac{m+1}{\lambda c} \alpha u^{\prime}+\left(K-\frac{\alpha^{2}}{4}\right) u \\
= & -\left[\left(p+\frac{1}{2}-K\right) u_{k}+\frac{1}{2 \lambda c}\left(\alpha^{2} u_{k}^{\prime \prime}+2(m+1) \alpha u_{k}^{\prime}\right)-B_{k+1} u_{0}\right] \\
& -\left(B_{1}+\cdots+B_{k+1}\right) u_{0} .
\end{aligned}
$$

La valeur de la constante $K$ est restée jusqu'ici indéterminée. Nous la choisirons de façon à annuler la somme

$$
B_{1}+B_{2}+\cdots+B_{k+1}
$$

de sorte que, finalement

$$
\begin{aligned}
& u^{\prime \prime}\left(1-\frac{\alpha^{2}}{2 \lambda c}\right)-\frac{m+1}{\lambda c} \alpha u^{\prime}+\left(K-\frac{\alpha^{2}}{4}\right) u \\
& =-\left(p+\frac{1}{2}-K\right) u_{k}-\frac{1}{2 \lambda c}\left[\alpha^{2} u_{k}^{\prime}+2(m+1) \alpha u_{k}^{\prime}\right]-B_{k+1} D_{p}(\alpha)
\end{aligned}
$$


On vérifie immédiatement que le second membre est une combinaison linéaire à coefficients constants des fonctions

$$
D_{p+4 k+4}, \cdots, D_{p+2}, D_{p-2}, \cdots, D_{p-4 k-4}
$$

ne contenant pas de terme en $D_{p}(\alpha)$.

La somme $B_{1}+B_{2}+\cdots+B_{k+1}$ a la forme suivante

$$
p+\frac{1}{2}-K+\frac{C_{3, p}}{2 \lambda c}+P\left(\frac{1}{\lambda c}, p+\frac{1}{2}-K\right)
$$

où $P$ est un polynôme de degré $k+1$ en $1 / \lambda c$ et $p+1 / 2-K$, ne contenant pas de termes du premier degré.

La valeur de $K$ qui nous intéresse est évidemment celle qui se réduit à $p+1 / 2$ lorsque $(\lambda c)^{-1} \rightarrow 0$. Pour $\lambda c$ assez grand, on pourra représenter cette valeur de $K$ par une série de puissances de $(\lambda c)^{-1}$, qui convergera certainement dès que $\lambda c$ dépassera une certaine valeur, de la forme suivante

$$
K=p+\frac{1}{2}+\frac{C_{3, p}}{2 \lambda c}+\frac{\gamma_{1}}{(2 \lambda c)^{2}}+\cdots
$$

où les $\boldsymbol{\gamma}$ sont des constantes.

Ceci peut encore s'écrire

$$
a=-\frac{1}{2}(\lambda c)^{2}+(2 p+1) \lambda c+C_{3, p}+\frac{\gamma_{1}}{2 \lambda c}+\cdots .
$$

Or $K$ étant une fonction analytique de $(\lambda c)^{-1}$, puisque la relation entre ces deux quantités est l'équation algébrique de degré $k+1$ (65), si, pour les valeurs de $\lambda c$ assurant la convergence de (66), on arrête le développement en série au terme en $(\lambda c)^{-k}$, le reste sera de la forme $O\left((\lambda c)^{-k-1}\right)$.

Il en résulte que si, dans le.second membre de (64), nous remplaçons partout $p+1 / 2-K$ par le développement en série limité au terme en $(\lambda c)^{-k}$, ce second membre deviendra une combinaison linéaire à coefficients constants des fonctions $D_{n+4 k+4}, \cdots, D_{n-4 k-4}$ dont tous les coefficients seront $O\left((\lambda c)^{-k-1}\right)$.

Or on sait que les fonctions paraboliques restent, pour toutes les valeurs réelles de la variable, inférieures à une certaine limite qui est fonction de l'indice de la fonction. Le second membre de (64) sera donc une fonction de $\alpha$ qui reste toujours inférieure à une certaine limite, dépendant de $p$ et de $k$, mais indépendante de $\alpha$.

L'équation (64) pourra donc s'écrire sous la forme

$$
u^{\prime \prime}\left(1-\frac{\alpha^{2}}{2 \lambda c}\right)+\frac{m+1}{\lambda c} \alpha u^{\prime}+\left(K-\frac{\alpha^{2}}{4}\right) u=O\left((\lambda c)^{-k-1}\right) .
$$

Si nous revenons maintenant à la variable $\mu=(2 \lambda c)^{1 / 2} \cdot \alpha$, on verra que 
$u\left((2 \lambda c)^{1 / 2} \cdot \alpha\right)$ vérifie l'équation suivante

$$
\left(1-\mu^{2}\right) u^{\prime \prime}-2(m+1) u^{\prime}+\left(a-m(m+1)-\lambda^{2} c^{2} \mu^{2}\right) u=O\left((\lambda c)^{-k}\right) .
$$

On sait d'autre part que l'équation (54) possède, pour certaines valeurs bien définies $a_{n}^{m}$ de la constante $a$, des solutions périodiques en $\eta$ de période $2 \pi$. Ces solutions se réduisent, pour $\lambda c=0$, aux fonctions associées de Legendre, et nous appellerons $P e_{r}^{m}(\cos \eta)$ ou $P e_{r}^{m}(\mu)$ la solution de (54) ou (55) qui, pour $\lambda c=0$, est égale à $P_{r}^{m}(\cos \eta)$ ou $P_{r}^{m}(\mu)$. Pour compléter la définition, nous imposerons de plus aux fonctions $P e_{r}^{m}$ de vérifier la condition de normalisation suivante

$$
\int_{-1}^{+1}\left[P e_{r}^{m}(\mu)\right]^{2} d \mu=\frac{r+m !}{r-m !} \cdot \frac{2}{2 r+1} .
$$

Les fonctions $P e_{r}^{m}(\mu)$ satisfont donc à l'équation différentielle

$$
\frac{d}{d \mu}\left[\left(1-\mu^{2}\right) \frac{d P e_{r}^{m}}{d \mu}\right]+\left(a_{r}^{m}-\lambda^{2} c^{2} \mu^{2}-\frac{m^{2}}{1-\mu^{2}}\right) P e_{r}^{m}=0 .
$$

On sait de plus que toute fonction de la forme $\left(1-\mu^{2}\right)^{m / 2} F(\mu)$, où $F(\mu)$ est une fonction bornée quelconque de $\mu$, peut, pour toutes les valeurs réelles de $\mu$ telles que $-1 \leqq \mu \leqq 1$, être développée en série convergente de fonctions $P e_{r}^{m}(\mu)$, chaque fois qu'elle peut l'être en série de fonctions $P_{r}^{m}(\mu)$.

Introduisons maintenant une nouvelle fonction $U(\mu)$ qui sera définie par

$$
U(\mu)=C\left(1-\mu^{2}\right)^{m / 2} u\left((2 \lambda c)^{1 / 2} \cdot \mu\right)
$$

où $C$ est une constante dont la valeur est donnée par la condition

$$
\int_{-1}^{1} U^{2}(\mu) d \mu=C^{2} \int_{-1}^{1} u^{2}\left((2 \lambda c)^{1 / 2} \cdot \mu\right)\left(1-\mu^{2}\right)^{m} d \mu=\frac{2}{2 n+1} \cdot \frac{n-m !}{n+m !}
$$

et $n$ étant un nombre entier qui sera déterminé plus loin. Cette fonction $U(\mu)$ vérifiera l'équation différentielle suivante

$$
\frac{d}{d \mu}\left[\left(1-\mu^{2}\right) \frac{d U}{d \mu}\right]+\left(a-\lambda^{2} c^{2} \mu^{2}-\frac{m^{2}}{1-\mu^{2}}\right) U=\phi(\mu)
$$

où $\phi(\mu)$ est une fonction bornée de $\mu$ telle que

$$
\phi(\mu)=O\left((\lambda c)^{-k}\right)
$$

La fonction $U(\mu)$ pourra évidemment être développée en série de fonctions $P_{e r}^{m}(\mu)$

$$
U(\mu)=\sum_{r=m}^{\infty} A_{r} P e_{r}^{m}(\mu)
$$


Il résulte de (69) et de (72), que

$$
\int_{-1}^{1} U^{2}(\mu) d \mu=\sum A_{r}^{2} \cdot \frac{2}{2 r+1} \cdot \frac{r+m !}{r-m !}=\frac{2}{2 n+1} \cdot \frac{n+m !}{n-m !} .
$$

D'autre part, $U(\mu)$ étant continu, ainsi que sa dérivée, dans tout le domaine $-1<\mu<1$ on aura aussi

$$
\frac{d}{d \mu}\left[\left(1-\mu^{2}\right) \frac{d U}{d \mu}\right]+\left(a-\lambda^{2} c^{2}-\frac{m^{2}}{1-\mu^{2}}\right) U=\sum A_{r}\left(a-a_{r}^{m}\right) P e_{r}^{m}=\phi(\mu) .
$$

Donc

$$
\begin{aligned}
\sum A_{r}^{2}\left(a-a_{r}^{m}\right)^{2} \cdot \frac{2}{2 r+1} \cdot \frac{r+m !}{r-m !} & =\int_{-1}^{1} \phi^{2}(\mu) d \mu \\
& =O\left((\lambda c)^{-2 k}\right) .
\end{aligned}
$$

Soit maintenant $\left(a-a_{n}^{m}\right)^{2}$ la plus petite des quantités, toutes différentes, $\left(a-a_{r}^{m}\right)^{2}$. L'entier $n$ correspondant sera celui à introduire dans la formule (72). Alors

$$
\sum A_{r}^{2}\left(a-a_{r}^{m}\right)^{2} \frac{2}{2 r+1} \cdot \frac{r+m !}{r-m !}>\left(a-a_{n}^{m}\right)^{2} \cdot \frac{2}{2 n+1} \cdot \frac{n-m !}{n+m !}
$$

et par conséquent

$$
a_{n}^{m}=a+O\left((\lambda c)^{-k}\right) .
$$

Il résulte de plus de (75), puisque, pour $r \neq n,\left(a-a_{r}^{m}\right)^{2}$ est une quantité finie, que

$$
A_{r}^{m}=O\left((\lambda c)^{-k}\right) \quad(r \neq n) .
$$

En introduisant ce dernier résultat dans (74), on voit ensuite que

$$
A_{n}^{m}=1+O\left((\lambda c)^{-2 k}\right) \text {. }
$$

De tout ceci il résulte enfin que

$$
U(\mu)=P e_{n}^{m}(\mu)+O\left((\lambda c)^{-k}\right)
$$

c'est à dire que, pour $\lambda c$ assez grand, $U(\mu)$ représente asymptotiquement une des fonctions $P e_{n}^{m}$, et $a$ la constante de séparation $a_{n}^{m}$ correspondante.

Il nous faut maintenant déterminer la valeur de l'entier $n$. Remarquons pour cela que, lorsque $\lambda c$ est assez grand, $U(\mu)$ diffère d'aussi peu qu'on veut de $\left(1-\mu^{2}\right)^{m / 2} D_{p}\left((2 \lambda c)^{1 / 2} \cdot \mu\right)$ et possède, lorsque $\mu$ varie entre -1 et +1 , (limites exclues), exactement $p$ zéros. On sait d'autre part, que la fonction $P e_{n}^{m}(\mu)$ possède, dans le même intervalle, $n-m$ zéros différents de $\mu= \pm 1$. 
Il en résulte donc que $U(\mu)$ sera une représentation asymptotique de $P e_{m+p}^{m}$, ou, en d'autres termes, que $p=n-m$.

En définitive, donc

$$
\begin{aligned}
U(\mu) & =P e_{m+p}^{m}(\mu)+O\left((\lambda c)^{-k}\right), \\
a & =a_{m+p}^{m}+O\left((\lambda c)^{-k}\right)
\end{aligned}
$$

avec $n=m+p$.

Nous allons maintenant donner les valeurs explicites de $U(\mu)$ et de $a$. On trouve que,

$$
u=u_{0}+u_{1}+u_{2}+\cdots
$$

avec

$$
\begin{aligned}
u_{0}(\alpha)= & D_{p}(\alpha), \\
u_{1}(\alpha)= & \frac{1}{2 \lambda c}\left[-\frac{1}{4} C_{1, p} D_{p+4}-\frac{1}{2} C_{2, p} D_{p+2}+\frac{1}{2} C_{4, p} D_{p-2}+\frac{1}{4} C_{6} D_{p-4}\right], \\
u_{2}(\alpha)= & \frac{1}{(2 \lambda c)^{2}}\left[\frac{1}{32} C_{1, p} C_{1, p+4} D_{p+8}+\frac{1}{24}\left(C_{1, p} C_{2, p+4}+2 C_{2, p} C_{1, p+2}\right) D_{p+6}\right. \\
& +\frac{1}{16}\left(C_{1, p} C_{3, p+4}+2 C_{2, p} C_{2, p+2}-C_{1, p} C_{3, p}\right) D_{p+4} \\
& +\frac{1}{8}\left(C_{1, p} C_{4, p+4}+2 C_{2, p} C_{3, p+2}-2 C_{4, p} C_{1, p-2}-2 C_{2, p} C_{3, p}\right) D_{p+2} \\
& +\frac{1}{8}\left(-2 C_{2, p} C_{5, p+2}+2 C_{4, p} C_{3, p-2}+C_{5, p} C_{2, p+4}-2 C_{3, p} C_{4, p}\right) D_{p-2} \\
& +\frac{1}{16}\left(2 C_{4, p} C_{4, p-2}+C_{6, p} C_{3, p-4}-C_{3, p} C_{5, p}\right) D_{p-4} \\
& \left.+\frac{1}{24}\left(2 C_{4, p} C_{5, p-2}+C_{5, p} C_{4, p-4}\right) D_{p-6}+\frac{1}{32} C_{5, p} C_{5, p-4} D_{p-8}\right] .
\end{aligned}
$$

Ensuite

$$
a=m(m+1)+(2 p+1) \lambda c+\gamma_{0}+\frac{\gamma_{1}}{2 \lambda c}+\frac{\gamma_{2}}{(2 \lambda c)^{2}}+\cdots
$$

avec

$$
\begin{aligned}
& \gamma_{0}=C_{8, p}, \\
& \gamma_{1}=-\frac{1}{4} C_{1, p} C_{5, p+4}-\frac{1}{2} C_{2, p} C_{4, p+2}+\frac{1}{2} C_{4, p} C_{2, p-2}+\frac{1}{4} C_{8, p} C_{1, p-4},
\end{aligned}
$$




$$
\begin{aligned}
\boldsymbol{\gamma}_{2}=\frac{1}{16} C_{5, p+4}\left(C_{1, p} C_{3, p+4}+2 C_{2, p} C_{2, p+2}-C_{1, p} C_{3, p}\right) \\
+\frac{1}{8} C_{4, p}\left(C_{1, p} C_{4, p+4}+2 C_{2, p} C_{3, p+8}-2 C_{4, p} C_{1, p-2}-2 C_{2, p} C_{3, p}\right) \\
\quad+\frac{1}{8} C_{2, p-2}\left(-2 C_{2, p} C_{5, p+2}+2 C_{4, p} C_{3, p-2}+C_{5, p} C_{2, p-4}-2 C_{3, p} C_{4, p}\right) \\
+\frac{1}{16} C_{1, p-4}\left(2 C_{4, p} C_{4, p-2}+C_{5, p} C_{3, p-4}-C_{3, p} C_{5, p}\right) .
\end{aligned}
$$

Pour faciliters l'emploi des ces formules, nous donnons ci-après une table des valeurs des coefficients $C$.

\begin{tabular}{|l|l|l|l|l|}
\hline$C_{1,0}=1 / 4$ & $C_{2,0}=-m$ & $C_{3,0}=-(m+3 / 4)$ & $C_{4,0}=0$ & $C_{5,0}=0$ \\
& & $C_{3,1}=-(m+7 / 4)$ & $C_{4,1}=0$ & $C_{5,1}=0$ \\
& & $C_{3,2}=-(m+15 / 4)$ & $C_{4,2}=2 m$ & $C_{5,2}=0$ \\
& & $C_{3,3}=-(m+27 / 4)$ & $C_{4,3}=6 m$ & $C_{5,3}=0$ \\
& & $C_{3,4}=-(m+43 / 4)$ & $C_{4,4}=12 m$ & $C_{5,4}=6$ \\
& & $C_{3,5}=-(m+63 / 4)$ & $C_{4,5}=20 m$ & $C_{5,5}=30$ \\
& & $C_{3,6}=-(m+87 / 4)$ & $C_{4,6}=30 m$ & $C_{5,6}=90$ \\
\hline
\end{tabular}

En effectuant les calculs, on trouve en particulier, en ne tenant pas compte des termes en $(\lambda c)^{-3}$

$p=0$

$$
\begin{aligned}
u=D_{0} & +\frac{1}{2 \lambda c}\left[-\frac{D_{4}}{16}+\frac{m}{2} D_{2}\right] \\
+ & \frac{1}{(2 \lambda c)^{2}}\left[\frac{D_{8}}{512}-\frac{m D_{6}}{32}+\frac{4 m^{2}-5}{32} D_{4}+\frac{9 m}{8} D_{2}\right] \\
a=\lambda c- & \frac{3}{4}+m^{2}-\left(\frac{3}{8}-m^{2}\right) \frac{1}{2 \lambda c}-\left(\frac{15}{16}-3 m^{2}\right) \frac{1}{(2 \lambda c)^{2}}
\end{aligned}
$$

$p=1$

$$
\begin{gathered}
u=D_{1}+\frac{1}{2 \lambda c}\left[-\frac{D_{5}}{16}+\frac{m D_{3}}{2}\right] \\
+\frac{1}{(2 \lambda c)^{2}}\left[\frac{D_{9}}{512}-\frac{m D_{7}}{32}+\frac{4 m^{2}-7}{32}+\frac{15 m}{8} D_{3}\right] \\
a=3 \lambda c-\frac{7}{4}+m^{2}-\left(\frac{15}{8}-3 m^{2}\right) \frac{1}{2 \lambda c}-\left(\frac{105}{16}-15 m^{2}\right) \frac{1}{(2 \lambda c)^{2}}
\end{gathered}
$$


$p=2$

$$
\begin{aligned}
& u=D_{2}+\frac{1}{2 \lambda c}\left[-\frac{D_{6}}{16}+\frac{m}{2} D_{4}+m D_{0}\right] \\
&+ \frac{1}{(2 \lambda c)^{2}}\left[\frac{D_{10}}{512}-\frac{m D_{8}}{32}+\frac{4 m^{2}-9}{32} D_{6}+\frac{41 m}{16} D_{4}+3 m D_{0}\right] \\
& a=5 \lambda c-\frac{15}{4}+m^{2}-\left(\frac{45}{8}-5 m^{2}\right) \frac{1}{2 \lambda c}-\left(\frac{405}{16}-39 m^{2}\right) \frac{1}{(2 \lambda c)^{2}}
\end{aligned}
$$

$p=3$

$$
\begin{aligned}
& u=D_{3}+\frac{1}{2 \lambda c}\left[-\frac{D_{7}}{16}+\frac{m D_{5}}{2}+3 m D_{1}\right] \\
& +\frac{1}{(2 \lambda c)^{2}}\left[\frac{D_{11}}{512}-\frac{m D_{9}}{32}+\frac{4 m^{2}-11}{32} D_{7}+\frac{51 m}{16} D_{5}+15 m D_{1}\right] \text {, } \\
& a=7 \lambda c-\frac{27}{4}+m^{2}-\left(\frac{105}{8}-7 m^{2}\right) \frac{1}{2 \lambda c}-\left(\frac{1155}{64}-75 m^{2}\right) \frac{1}{(2 \lambda c)^{2}} .
\end{aligned}
$$

Il nous reste maintenant à déterminer la valeur de la constante de normalisation $C$. Nous avons vu que celle-ci est définie par la relation suivante

$$
\frac{C^{2}}{(2 \lambda c)^{1 / 2}} \int_{-(2 \lambda c)^{1 / 2}}^{(2 \lambda c) 1 / 2} u^{2}(\alpha)\left(1-\frac{\alpha^{2}}{2 \lambda c}\right)^{m} d \alpha=\frac{2}{2 n+1} \cdot \frac{n+m !}{n-m !} .
$$

Dans l'intégrale on pourra évidemment remplacer $\left(1-\alpha^{2} / 2 \lambda c\right)^{m}$ par son développement. En arrêtant celui-ci au terme en $(\lambda c)^{-k}$, le reste sera $O\left((\lambda c)^{-k-1}\right)$.

L'expression à intégrer sera alors égale au produit d'un polynôme en $\alpha^{2}$ par $e^{-\alpha^{2} / 2}$. Par conséquent si, pour $\lambda c$ assez grand, au lieu d'intégrer entre $-(2 \lambda c)^{1 / 2}$ et $(2 \lambda c)^{1 / 2}$, on intègre entre $-\infty$ et $\infty$, l'erreur commise sera de la forme $O\left(e^{-\lambda c /(2 \lambda c)^{1 / 2}}\right)$ et nous n'aurons donc pas à en tenir compte. Dans ces conditions, l'équation (84) devient

$$
\begin{aligned}
\frac{C^{2}}{(2 \lambda c)^{1 / 2}} \int_{-\infty}^{\infty}\left(u_{0}+u_{1}+u_{2}+\cdots\right)^{2} \\
\cdot\left(1-m \frac{\alpha^{2}}{2 \lambda c}+\frac{m(m-1)}{2} \cdot \frac{\alpha^{4}}{(2 \lambda c)^{2}}-\cdots\right) d \alpha=\frac{2}{2 n+1} \cdot \frac{n+m !}{n-m !} .
\end{aligned}
$$

L'intégrale se calcule facilement et a pour valeur 


$$
(2 \pi)^{1 / 2} \cdot p !\left(c_{0}+\frac{c_{1}}{2 \lambda c}+\frac{c_{2}}{(2 \lambda c)^{2}}+\cdots\right)
$$

avec

$$
\begin{aligned}
c_{0}= & 1, \quad c_{1}=-\frac{m}{2}(2 p+1), \\
c_{2}= & \frac{(p+1)(p+2)(p+3)(p+4)+p(p-1)(p-2)(p-3)}{256} \\
& -3 m^{2} \frac{(p+1)(p+2)+p(p-1)}{4}+3 m(m-1) \cdot \frac{2 p^{2}+2 p+1}{2} .
\end{aligned}
$$

Finalement, donc

$$
C=\frac{1}{p !}\left(\frac{4 \lambda c}{\pi}\right)^{1 / 4}\left(\frac{p+2 m !}{2 p+2 m+1}\right)^{1 / 2} /\left(c_{0}+\frac{c_{1}}{2 \lambda c}+\frac{c_{2}}{(2 \lambda c)^{2}}+\cdots\right)^{1 / 2} .
$$

En particulier

$p=0$

$$
C=\left(\frac{4 \lambda c}{\pi}\right)^{1 / 4}\left(\frac{2 m !}{2 m+1}\right)^{1 / 2} /\left[1-\frac{m}{2} \frac{1}{2 \lambda c}+3\left(\frac{1}{32}-\frac{m}{2}\right) \cdot \frac{1}{(2 \lambda c)^{2}}\right]^{1 / 2} .
$$

$p=1$

$$
\begin{aligned}
C= & \left(\frac{4 \lambda c}{\pi}\right)^{1 / 4} \\
& \cdot\left(\frac{2 m+1 !}{2 m+3}\right)^{1 / 2} /\left[1-\frac{3 m}{2} \cdot \frac{1}{2 \lambda c}+\left(\frac{15}{32}-\frac{15 m}{2}+\frac{6 m^{2}}{2}\right) \frac{1}{(2 \lambda c)^{2}}\right]^{1 / 2} .
\end{aligned}
$$

$p=2$

$$
\begin{aligned}
C=\frac{1}{2} & \cdot\left(\frac{4 \lambda c}{\pi}\right)^{1 / 4} \\
& \cdot\left(\frac{2 m+2 !}{2 m+5}\right)^{1 / 2} /\left[1-\frac{5 m}{2} \cdot \frac{1}{2 \lambda c}+\left(\frac{45}{32}-\frac{39 m}{2}+9 m^{2}\right) \frac{1}{(2 \lambda c)^{2}}\right]^{1 / 2} .
\end{aligned}
$$

$p=3$

$$
\begin{aligned}
C= & \frac{1}{6} \cdot\left(\frac{4 \lambda c}{\pi}\right)^{1 / 4} \\
& \cdot\left(\frac{2 m+3 !}{2 m+7}\right)^{1 / 2} /\left[1-\frac{7 m}{2} \cdot \frac{1}{2 \lambda c}+\left(\frac{105}{32}-\frac{75 m}{2}+18 m^{2}\right) \frac{1}{(2 \lambda c)^{2}}\right]^{1 / 2} .
\end{aligned}
$$

Il est intéressant de comparer les valeurs que fournissent ces formules 
asymptotiques avec les valeurs exactes des fonctions $P e_{n}^{m}$ et des constantes de séparation $a_{n}^{m}$ correspondantes.

A notre connaissance, les seules tables numériques publiées jusqu'ici relatives aux fonctions sphéroidales sont celles calculées par Stratton, Morse, Chu et Hutner. Ces auteurs donnent, pour les valeurs de $\lambda c$ comprises entre 0 et 5 , et variant de 0,1 en 0,1 , les valeurs des constantes de séparation et des coefficients du développement de la fonction $P e_{n}^{m}$ en série de fonctions associées de Legendre. Toutefois, comme Stratton et al utilisent, pour normaliser leurs fonctions, une convention différente de la notre, notamment la condition $P e_{n}^{m}(0)=1$, pour $n-m$ pair, et $(d / d \mu) P e_{n}^{m}(0)=1$, pour $n-m$ impair, il faudra tenir compte de cette différence avant de faire la comparaison.

D'autre part, il est évident, si on se rapelle les résultats obtenus pour les fonctions de Mathieu, que la valeur la plus élevée de $\lambda c$, c'est à dire $\lambda c=5$, contenue dans les tables de Stratton et al, est un peu faible pour espérer une très bonne approximation.

Voici les valeurs obtenues

\begin{tabular}{|l|l|l|r|r|}
\hline \multicolumn{5}{|c|}{$\lambda c=5$} \\
\hline$p$ & $m$ & $n$ & $a$ approximatif & $a$ exact \\
\hline 0 & 0 & 0 & 4,2031 & 4,1951 \\
0 & 1 & 1 & 5,3336 & 5,3504 \\
0 & 2 & 2 & 8,8138 & 8,7477 \\
0 & 3 & 3 & 14,3731 & 14,3098 \\
1 & 0 & 1 & 12,9518 & 12,9117 \\
1 & 1 & 2 & 14,6629 & 14,6429 \\
2 & 0 & 2 & 20,1886 & 20,1769 \\
2 & 1 & 3 & 22,6630 & 23,3976 \\
3 & 0 & 3 & 25,2722 & 26,5873 \\
\hline
\end{tabular}

On voit que l'approximation, compte tenu de la simplicité des formules et de la valeur peu élevée de $\lambda c$, est tout à fait satisfaisante pour les faibles valeurs de $n$ et $m$. Les formules deviennent de plus en plus inexactes au fur et à mesure que $n$ ou $m$ croit.

Passons maintenant aux fonctions $P e_{n}^{m}$ elles mêmes. Nous avons calculé, pour $P e_{0}, P e_{2}$ et $P e_{1}^{1}$, et $\lambda c=5$, les valeurs exactes correspondant à $\mu=0$, ainsi que les valeurs approchées, pour la même valeur de $\mu$, résultant des formules données plus haut, soit:

$$
P e_{0}(0) \sim\left(\frac{4 \lambda c}{\pi}\right)^{1 / 4}\left(1-\frac{3}{32 \lambda c}-\frac{135}{2048(\lambda c)^{2}}\right) /\left(1+\frac{3}{128(\lambda c)^{2}}\right)^{1 / 2},
$$




$$
\begin{aligned}
-P e_{2}(0) & \sim\left(\frac{\lambda c}{25 \pi}\right)^{1 / 4}\left(1-\frac{15}{32 \lambda c}-\frac{1215}{2048(\lambda c)^{2}}\right) /\left(1+\frac{45}{128(\lambda c)^{2}}\right)^{1 / 2}, \\
P e_{1}^{1}(0) \sim\left(\frac{16 \lambda c}{9 \pi}\right)^{1 / 4} & \\
& \cdot\left(1-\frac{11}{32 \lambda c}-\frac{279}{2048(\lambda c)^{2}}\right) /\left(1-\frac{1}{4 \lambda c}-\frac{45}{128(\lambda c)^{2}}\right)^{1 / 2} .
\end{aligned}
$$

Voici les résultats obtenus

\begin{tabular}{|c|c|c|}
\hline \multicolumn{3}{|c|}{$\lambda c=5$} \\
\hline & Valeur approximative & Valeur exacte \\
\hline$P e_{0}(0)$ & 1,5537 & 1,5505 \\
$P e_{2}(0)$ & $-0,4371$ & $-0,4272$ \\
$P e_{1}^{1}(0)$ & 1,2411 & 1,2662 \\
\hline
\end{tabular}

Comme dans le cas des constantes de séparation, on constate que l'approximation est bonne pour $n=0$ et devient de plus en plus mauvaise au fur et à mesure que $n$ et $m$ augmentent. L'exactitude de nos formules, étant donné leur simplicité, est remarquable dans le cas de $n=0$. Pour obtenir la même précision, dans les autres cas, il faudrait tenir compte d'un nombre de plus en plus grand de termes, au fur et à mesure que $n$ et $m$ augmentent, dans le développement asymptotique.

Nos formules asymptotiques nous donnent également des renseignements intéressants sur le comportement des fonctions $P e_{n}^{m}(\mu)$ lorsque $\lambda c$ devient très grand.

On voit que, par suite de la présence du facteur $e^{-2^{-1} \lambda c \cos ^{2} \eta}$, si l'on fait croître $\lambda c$ indéfiniment en maintenant $n$ et $m$ constants, la fonction $P e_{n}^{m}(\mu)$ tendra uniformément vers zéro pour toutes les valeurs de $\mu$ différentes de zéro.

Les $n-m$ zéros, différents de $\mu=0$, que la fonction possède entre 0 et 1 set rapprochent, lorsque $\lambda c$ croît indéfiniment, de $\mu=0$, et il en est de même des maxima et des minima qui les séparent.

Pour $\mu=0$, lorsque $n-m$ est pair, la fonction possède un maximum ou un minimum qui croit indéfiniment avec $\lambda c$, mais de telle manière cependant que

$$
\lim _{\lambda c \rightarrow \infty} \frac{P e_{n}^{m}(0)}{(\lambda c)^{1 / 4}}=(-1)^{(n-m) / 2}\left(\frac{4}{\pi}\right)^{1 / 4} \frac{(n+m !)^{1 / 2}}{(2 n+1)^{1 / 2} \cdot\left(\frac{n-m}{2} !\right)} .
$$

Lorsque $n-m$ est impair, la fonction est évidemment nulle pour $\mu=0$, 
mais la dérivée en ce point devient infinie en même temps que $\lambda c$, de telle manière que

$$
\lim _{\lambda c \rightarrow \infty} \frac{P e_{n}^{\prime m}(0)}{(\lambda c)^{3 / 4}}=(-1)^{(n-m-1) / 2}\left(\frac{16}{\pi}\right)^{1 / 4} \frac{(n+m !)^{1 / 2}}{(2 n+1)^{1 / 2} \cdot\left(\frac{n-m-1}{2} !\right)} .
$$

2. Fonctions d'onde sphéroidales. B. Ellipsoide aplati. Dans l'équation des fonctions d'onde sphéroidales associées à l'ellipsoide de révolution aplati

$$
\frac{1}{\cos \eta} \cdot \frac{d}{d \eta}\left(\cos \eta \frac{d H}{d \eta}\right)+\left(A-\lambda^{2} c^{2} \cos ^{2} \eta-\frac{m^{2}}{\cos ^{2} \eta}\right) H=0
$$

faisons d'abord $\mu_{1}=\cos \eta$, ce qui la transforme en

$$
\left(1-\mu_{1}^{2}\right) \frac{d^{2} H}{d \mu_{1}^{2}}+\left(\frac{1}{\mu_{1}}-2 \mu_{1}\right) \frac{d H}{d \mu_{1}}+\left(A-\lambda^{2} c^{2} \mu_{1}^{2}-\frac{m^{2}}{\mu_{1}^{2}}\right) H=0 .
$$

Changeons ensuite une seconde fois de variable indépendante en posant $\mu_{1}=\alpha(2 \lambda c)^{1 / 2}$. L'équation prend alors la forme suivante

$$
\left(1-\frac{\alpha^{2}}{2 \lambda c}\right) \frac{d^{2} H}{d \alpha^{2}}+\left(\frac{1}{\alpha}-\frac{\alpha}{2 \lambda c}\right) \frac{d H}{d \alpha}+\left(\frac{A}{2 \lambda c}-\frac{\alpha^{2}}{4}-\frac{m^{2}}{\alpha^{2}}\right) H=0 .
$$

Nous allons maintenant former une suite de fonctions $u_{0}, u_{1}, u_{2}, \cdots, u_{k}$ de $\alpha$ qui vérifient les équations différentielles suivantes

$$
\begin{aligned}
& u_{0}^{\prime \prime}+\frac{1}{\alpha} u_{0}^{\prime}+\left(m+2 p+1-\frac{\alpha^{2}}{4}-\frac{m^{2}}{\alpha^{2}}\right) u_{0}=0 \\
& u_{1}^{\prime \prime}+\frac{1}{\alpha} u_{1}^{\prime}+\left(m+2 p+1-\frac{\alpha^{2}}{4}-\frac{m^{2}}{\alpha^{2}}\right) u_{1} \\
& =\left(m+2 p+1-\frac{A}{2 \lambda c}\right) u_{0}+\frac{1}{2 \lambda c}\left(\alpha^{2} u_{0}^{\prime \prime}+2 \alpha u_{0}^{\prime}\right)-B_{1} u_{0} \\
& u_{k}^{\prime \prime}+\frac{1}{\alpha} u_{k}^{\prime}+\left(m+2 p+1-\frac{\alpha^{2}}{4}-\frac{m^{2}}{\alpha^{2}}\right) u_{k} \\
& \quad=\left(m+2 p+1-\frac{A}{2 \lambda c}\right) u_{k-1}+\frac{1}{2 \lambda c}\left(\alpha^{2} u_{k-1}^{\prime \prime}+2 \alpha u_{k-1}^{\prime}\right)-B_{k} u_{0} .
\end{aligned}
$$

Dans ces équations, $p$ est un nombre entier positif quelconque, et $B_{1}, \cdots, B_{k}$ sont des constantes qui seront définies plus loin.

Comme valeur de $u_{0}(\alpha)$ nous prendrons

$$
u_{0}(\alpha)=\alpha^{m} e^{-\alpha^{2} / 4} L_{p}^{(m)}\left(\alpha^{2} / 2\right)
$$


où $L_{p}^{(m)}(x)$ représente le polynôme de Laguerre généralisé défini par

$$
L_{p}^{(m)}(x)=\frac{1}{p !} x^{-m} e^{x} \frac{d^{p}}{d x^{p}}\left(x^{m+p} e^{-x}\right) .
$$

D'après les propriétés des polynômes $L_{p}^{(m)}$, on vérifie immédiatement qu'on a l'identité suivante

$$
\begin{aligned}
& \left(\alpha^{2} \frac{d^{2}}{d \alpha^{2}}+2 \alpha \frac{d}{d \alpha}\right) \alpha^{m} e^{-\alpha^{2} / 4} L_{p}^{(m)}\left(\frac{\alpha^{2}}{2}\right) \\
& \quad=\alpha^{m} e^{-\alpha^{2} / 4}\left(C_{1, p} L_{p+2}^{(m)}+C_{2, p} L_{p+1}^{(m)}+C_{3, p} L_{p}^{(m)}+C_{4, p} L_{p-1}^{(m)}+C_{5, p} L_{p-2}^{(m)}\right)
\end{aligned}
$$

avec

$$
\begin{aligned}
& C_{1, p}=(p+1)(p+2), \quad C_{2, p}=-(p+1), \\
& C_{3, p}=-\left(2 p^{2}+2 p m+2 p+m+1\right), \quad C_{4, p}=p+m, \\
& C_{5, p}=(p+m)(p+m-1) .
\end{aligned}
$$

Bien entendu, dans cette formule, les fonctions d'indice négatif doivent être considérées comme identiquement nulles.

On pourra donc mettre le second membre de l'équation (921) sous la forme suivante

$$
\begin{aligned}
& (1 / 2 \lambda c) \alpha^{m} e^{-\alpha^{2} / 4}\left\{C_{1, p} L_{p+2}+C_{2, p} L_{p+1}\right. \\
& \left.+\left[2 \lambda c\left(m+2 p+1-B_{1}\right)-A+C_{3, p}\right] L_{p}+C_{4, p} L_{p-1}+C_{5, p} L_{p-2}\right\} .
\end{aligned}
$$

Nous choisirons la valeur de $B_{1}$ de façon à annuler le coefficient de $L_{p}^{(m)}\left(\alpha^{2} / 2\right)$, et nous prendrons comme solution de l'équation $\left(92_{1}\right)$ une combinaison linéaire à coefficients constants des fonctions $L_{p+2}^{(m)}, L_{p+1}^{(m)}, L_{p-1}^{(m)}, L_{p-2}^{(m)}$ multipliées $\operatorname{par} \alpha^{m} e^{-\alpha^{2} / 4}$. Nous trouverons ainsi

$$
\begin{aligned}
u_{1}(\alpha) & =\frac{1}{2 \lambda c} \alpha^{m} e^{-\alpha^{2} / 4}\left[-\frac{1}{4} C_{1, p} L_{p+2}^{(m)}-\frac{1}{2} C_{2, p} L_{p+1}^{(m)}+\frac{1}{2} C_{4, p} L_{p-1}^{(m)}+\frac{1}{4} C_{5, p} L_{p-2}^{(m)}\right], \\
B_{1} & =m+2 p+1-\frac{A}{2 \lambda c}+\frac{C_{3, p}}{2 \lambda c} .
\end{aligned}
$$

Les valeurs de $u_{2}, u_{3}$ s'obtiendrons de la même manière. $u_{k}(\alpha)$ sera donc une combinaison linéaire à coefficients constants, multipliée par $\alpha^{m} e^{-\alpha^{2} / 4}$, des fonctions $L_{p+2 k}^{(m)}, L_{p+2 k-1}^{(m)}, \cdots, L_{p+1}^{(m)}, L_{p-1}^{(m)}, \cdots, L_{p-2 k}^{(m)}$, ne contenant pas de terme en $\alpha^{m} e^{-\alpha^{2} / 4} L_{p}^{(m)}$.

Additionnons maintenant membre à membre les équations $\left(92_{0}\right),\left(92_{1}\right), \cdots$, $\left(92_{k}\right)$, et ajoutons aux deux membres la quantité

$$
\left(m+2 p+1-\frac{A}{2 \lambda c}\right) u_{k}+\frac{1}{2 \lambda c}\left(\alpha^{2} u_{k}^{\prime \prime}+\alpha u_{k}^{\prime}\right)-B_{k+1} u_{0},
$$


$B_{k+1}$ étant choisi de façon à annuler le coefficient de $u_{0}$. En posant

$$
u=u_{0}+u_{1}+\cdots+u_{k}
$$

on obtient ainsi

$$
\begin{aligned}
u^{\prime \prime}+\frac{1}{\alpha} u^{\prime}+(m+ & \left.2 p+1-\frac{\alpha^{2}}{4}-\frac{m^{2}}{\alpha^{2}}\right) u \\
= & \left(m+2 p+1-\frac{A}{2 \lambda c}\right) u+\frac{1}{2 \lambda c}\left(\alpha^{2} u^{\prime \prime}+\alpha u^{\prime}\right)+B_{k+1} u_{0} \\
& -\left[\left(m+2 p+1-\frac{A}{2 \lambda c}\right) u_{k}+\frac{1}{2 \lambda c}\left(\alpha^{2} u_{k}^{\prime \prime}+\alpha u_{k}^{\prime}\right)\right] \\
& -\left(B_{1}+B_{2}+\cdots+B_{k+1}\right) \alpha^{m} e^{-\alpha^{2} / 4} L_{p}^{(m)} .
\end{aligned}
$$

Cette équation peut encore s'écrire

$$
\begin{aligned}
u^{\prime \prime}\left(1-\frac{\alpha^{2}}{2 \lambda c}\right)+u^{\prime}\left(\frac{1}{\alpha}-\frac{\alpha}{2 \lambda c}\right)+\left(\frac{A}{2 \lambda c}-\frac{\alpha^{2}}{4}-\frac{m^{2}}{\alpha^{2}}\right) u \\
=-\left[\left(m+2 p+1-\frac{A}{2 \lambda c}\right) u_{k}+\frac{1}{2 \lambda c}\left(\alpha^{2} u_{k}^{\prime \prime}+\alpha u_{k}^{\prime}\right)\right. \\
\left.\quad-B_{k+1} \alpha^{m} e^{-\alpha^{2} / 4} L_{p}^{(m)}\right]-\left(B_{1}+\cdots+B_{k+1}\right) \alpha^{m} e^{-\alpha^{2} / 4} L_{p}^{(m)} .
\end{aligned}
$$

Nous choisirons maintenant la valeur de la constante $A$, qui est restée jusqu'ici indéterminée, de façon à avoir

$$
B_{1}+B_{2}+\cdots+B_{k+1}=0
$$

de sorte que $u(\alpha)$ vérifiera finalement l'équation suivante

$$
\begin{aligned}
u^{\prime \prime}\left(1-\frac{\alpha^{2}}{2 \lambda c}\right)+ & u^{\prime}\left(\frac{1}{\alpha}-\frac{\alpha}{2 \lambda c}\right)+\left(\frac{A}{2 \lambda c}-\frac{\alpha^{2}}{4}-\frac{m^{2}}{\alpha^{2}}\right) u \\
=-\left[\left(m+2 p+1-\frac{A}{2 \lambda c}\right) u_{k}\right. & +\frac{1}{2 \lambda c}\left(\alpha^{2} u_{k}^{\prime \prime}+\alpha u_{k}^{\prime}\right) \\
& \left.-B_{k+1} \alpha^{m} e^{-\alpha^{2} / 4} L_{p}^{(m)}\right] .
\end{aligned}
$$

La somme $B_{1}+B_{2}+\cdots+B_{k+1}$ a la forme suivante

$$
m+2 p+1-\frac{A}{2 \lambda c}+\frac{C_{8, p}}{2 \lambda c}+P
$$

où $P$ représente un polynôme de degré $k+1$ en $(m+2 p+1-A / 2 \lambda c)$ et $(\lambda c)^{-1}$, 
ne contenant pas de termes du premier degré.

La valeur de $A / 2 \lambda c$ qui nous intéresse est évidemment celle qui se réduit à $(m+2 p+1)$ lorsque $\lambda c$ croit indéfiniment.

Pour $\lambda c$ suffisamment grand, on peut représenter cette valeur de $A / 2 \lambda c$ au moyen d'une série procédant suivant les puissances décroissantes de $\lambda c$. Ce développement, convergent dès que $\lambda c$ est assez grand, aura la forme suivante

$$
\frac{A}{2 \lambda c}=m+2 p+1+\frac{C_{3, p}}{2 \lambda c}+\frac{\gamma_{1}}{(2 \lambda c)^{2}}+\cdots .
$$

$A$ étant une fonction analytique de $(\lambda c)^{-1}$, si, pour les valeurs de $(\lambda c)^{-1}$ assurant la convergence, on arrête le développement au terme en $(\lambda c)^{-k}$, le reste sera de la forme $O\left((\lambda c)^{-(k+1)}\right)$.

Il en résulte que, si, dans le second membre de (96), nous remplaçons partout $m+2 p+1-A / 2 \lambda c$ par le développement en série (98), ce second membre deviendra une combinaison linéaire à coefficients constants des fonctions $L_{p+2 k+2}^{(m)}, \cdots, L_{p+1}^{(m)}, L_{p-1}^{(m)}, \cdots, L_{p-2 k-2}^{(m)}$, multipliée par $\alpha^{m} e^{-\alpha^{2} / 4}$, dans laquelle tous les coefficients constants sont $O\left((\lambda c)^{-(k+1)}\right)$.

Or les fonctions $\alpha^{m} e^{-\alpha^{2} / 4} L_{p}^{(m)}\left(\alpha^{2} / 2\right)$ restent, lorsque $\alpha$ a une valeur réelle quelconque, inférieures à une certaine limite, fonction de $p$, mais indépendante de $\alpha$. Par conséquent, le second membre de l'équation (96) sera, lui aussi, inférieur, quel que soit $\alpha$, à une certaine limite, fonction seulement de $p$ et de $k$.

L'équation (96) pourra donc s'écrire de la manière suivante

$$
u^{\prime \prime}\left(1-\frac{\alpha^{2}}{2 \lambda c}\right)+u^{\prime}\left(\frac{1}{\alpha}-\frac{\alpha}{2 \lambda c}\right)+\left(\frac{A}{2 \lambda c}-\frac{\alpha^{2}}{4}-\frac{m^{2}}{\alpha^{2}}\right) u=O\left((\lambda c)^{-k-1}\right) .
$$

En revenant maintenant à la variable $\mu_{1}$, on voit de suite que $u\left((2 \lambda c)^{1 / 2} \cdot \mu_{1}\right)$ vérifiera l'équation différentielle

$$
\left(1-\mu_{1}^{2}\right) \frac{d^{2} u}{d \mu_{1}^{2}}+\left(\frac{1}{\mu_{1}}-2 \mu_{1}\right) \frac{d u}{d \mu_{1}}+\left(A-\lambda^{2} c^{2} \mu_{1}^{2}-\frac{m^{2}}{\mu_{1}^{2}}\right) u=O\left((\lambda c)^{-k}\right)
$$

Dans ce qui va suivre, nous prendrons comme variable indépendante, au lieu de $\mu_{1}$, une quantité $\mu=\sin \eta$ définie par la relation

$$
\mu_{1}^{2}+\mu^{2}=1 \text {. }
$$

L'équation (100) prendra alors la forme

$$
\frac{d}{d \mu}\left[\left(1-\mu^{2}\right) \frac{d u}{d \mu}\right]+\left(b+\lambda^{2} c^{2} \mu^{2}-\frac{m^{2}}{1-\mu^{2}}\right) u=O\left((\lambda c)^{-k}\right)
$$

où $u$ représente $u\left(\left(2 \lambda c\left(1-\mu^{2}\right)\right)^{1 / 2}\right)$, et où l'on a posé $b=A-\lambda^{2} c^{2}$. Avec le même changement de variable, l'équation initiale des ondes sphéroidales 
aplaties devient identique à l'équation des ondes sphéroidales allongées (55), avec cette différence toutefois que le coefficient de $(\lambda c)^{2}$ est maintenant positif au lieu d'être négatif. Comme nous le verrons, ce changement de signe modifie complètement le comportement asymptotique des solutions pour $\lambda c$ très grand.

On sait que l'équation (89) des fonctions sphéroidales aplaties ne possède de solutions périodiques en $\eta$, de période $2 \pi$, que pour certaines valeurs bien définies de la constante $b$. Pour $\lambda c=0$, ces solutions se réduisent aux fonctions sphériques associées $P_{n}^{m}(\sin \eta)=P_{n}^{m}(\mu)$, et nous appellerons $\bar{P} e_{n}^{m}(\mu)$ la solution périodique (en $\eta)$ de (89) qui, pour $\lambda c=0$, est identique à $P_{n}^{m}(\sin \eta)$, et la valeur correspondante de $b$ sera représentée par $b_{n}^{m}$. Pour compléter la définition de $\bar{P} e_{n}^{m}$, nous lui imposerons de plus de satisfaire à la condition de normalisation suivante

$$
\int_{-1}^{1}\left[\bar{P} e_{n}^{m}(\mu)\right]^{2} d \mu=\frac{2}{2 n+1} \cdot \frac{n+m !}{n-m !} .
$$

Toute comme dans le cas des fonctions $P e_{n}^{m}(\mu)$, toute fonction de la forme $\left(1-\mu^{2}\right)^{m / 2} F(\mu)$, où $F(\mu)$ est une fonction bornée quelconque, pourra être développée en série convergente de fonctions $\bar{P} e_{n}^{m}(\mu)$ dans le domaine réel $-1 \leqq \mu \leqq 1$, chaque fois qu'elle pourra l'être en série de fonctions sphériques associées $P_{n}^{m}(\mu)$. Introduisons maintenant une nouvelle fonction $U(\mu)$ qui sera définie par

$$
U(\mu)=C u\left(\left(2 \lambda c\left(1-\mu^{2}\right)\right)^{1 / 2}\right)
$$

où $C$ est une constante telle que

$$
\int_{-1}^{1} U^{2}(\mu) d \mu=C^{2} \int_{-1}^{1} u^{2}\left(\left(2 \lambda c\left(1-\mu^{2}\right)\right)^{1 / 2}\right) d \mu=\frac{2}{2 n+1} \cdot \frac{n+m !}{n-m !} .
$$

Cette fonction $U(\mu)$ satisfaira évidemment à l'équation différentielle suivante

$$
\frac{d}{d \mu}\left[\left(1-\mu^{2}\right) \frac{d U}{d \mu}\right]+\left(b+\lambda^{2} c^{2} \mu^{2}-\frac{m^{2}}{1-\mu^{2}}\right) U=\phi(\mu)
$$

où $\phi(\mu)$ est une fonction bornée de $\mu$ telle que, pour $\lambda c$ très grand

$$
\phi(\mu)=O\left((\lambda c)^{-k}\right) \text {. }
$$

La fonction $U(\mu)$, étant paire et continue, ainsi que sa dérivée, pour $-1 \leqq \mu \leqq 1$, pourra être développée en série convergente, deux fois dérivable, de fonctions $\bar{P} e_{n}^{m}(\mu)$ paires, sous la forme

$$
U(\mu)=\sum_{r=m}^{\infty} A_{r} \bar{P} e_{r}^{m}(\mu)
$$


et il résulte de (103) et de (105) que

$$
\int_{-1}^{1} U^{2}(\mu) d \mu=\sum A_{r}^{2} \cdot \frac{2}{2 r+1} \cdot \frac{r+m !}{r-m !}=\frac{2}{2 n+1} \cdot \frac{n+m !}{n-m !} .
$$

D'autre part, $\phi(\mu)$ pouvant lui aussi être développé en série convergente de fonctions $\bar{P} e_{n}^{m}$ on aura, en vertu de (104)

$$
\phi(\mu)=\sum_{r=m}^{\infty} A_{r}\left(b-b_{r}^{m}\right) \bar{P} e_{r}^{m}(\mu)
$$

d'où

$$
\int_{-1}^{1} \phi^{2}(\mu) d \mu=\sum \frac{2}{2 r+1} \cdot \frac{r+m !}{r-m !} \cdot A_{r}^{2}\left(b-b_{r}^{m}\right)^{2}=O\left((\lambda c)^{-2 k}\right) .
$$

Nous choisirons l'entier $n$, resté jusqu'ici indéterminé, de façon à ce que $\left(b-b_{n}^{m}\right)^{2}$ soit la plus petite des quantités, toutes différentes, $\left(b-b_{r}^{m}\right)^{2}$. Alors

$$
\sum \frac{2}{2 r+1} \cdot \frac{r+m !}{r-m !} \cdot A_{r}^{2}\left(b-b_{r}^{m}\right)^{2}>\left(b-b_{n}^{m}\right)^{2} \frac{2}{2 n+1} \cdot \frac{n+m !}{n-m !},
$$

et par conséquent

$$
b=b_{n}^{m}+O\left((\lambda c)^{-k}\right) .
$$

Comme, d'autre part, toutes les autres quantités $\left(b-b_{r}^{m}\right)^{2}$ sont finies, on aura nécessairement

$$
A_{r}=O\left((\lambda c)^{-k}\right)
$$

pour $r \neq n$, tandis que, pour $r=n$, on aura

$$
A_{r}=1+O\left((\lambda c)^{-2 k}\right) \text {. }
$$

De tout ceci, il résulte enfin que

$$
U(\mu)=\bar{P} e_{n}^{m}(\mu)+O\left((\lambda c)^{-k}\right)
$$

c'est à dire que $U(\mu)$ représente asymptotiquement une des fonctions $\bar{P} e_{n}^{m}(\mu)$ paires, et que $b$ représente asymptotiquement la constante de séparation correspondante.

Il nous faut donc maintenant déterminer la relation existant entre $n$ et $p$, c'est à dire rechercher laquelle des fonctions $\bar{P} e_{n}^{m}(\mu)$ est représentée asymptotiquement par $U(\mu)$.

Lorsque $\lambda c$ est assez grand, $U(\mu)$ diffère d'aussi peu qu'on veut de

$$
C(2 \lambda c)^{m / 2}\left(1-\mu^{2}\right)^{m / 2} e^{-\lambda c\left(1-\mu^{2}\right) / 2} L_{p}^{(m)}\left[\lambda c\left(1-\mu^{2}\right)\right]
$$

et possède, par conséquent, lorsque $-1<\mu<1$, exactement $2 p$ zéros différents 
de $\mu= \pm 1$. D'autre part, lorsque $n-m$ est pair, la fonction $\bar{P} e_{n}^{m}(\mu)$ possède, dans le même domaine, $n-m$ zéros différents de $\mu= \pm 1$. Par conséquent, on aura donc

c'est à dire enfin

$$
p=\frac{(n-m)}{2}
$$

$$
\begin{aligned}
U(\mu) & =\bar{P} e_{n}^{m}(\mu)+O\left((\lambda c)^{-k}\right), \\
b & =b_{n}^{m}+O\left((\lambda c)^{-k}\right)
\end{aligned}
$$

avec $p=(n-m) / 2$.

Passons maintenant aux fonctions $\bar{P} e_{n}^{m}(\mu)$ impaires. Considérons tout d'abord une fonction $v_{1}(\mu)$ égale à $u\left(\left(2 \lambda c\left(1-\mu^{2}\right)\right)^{1 / 2}\right)$ pour $0<\mu<1$ et à $-u\left(\left(2 \lambda c\left(1-\mu^{2}\right)\right)^{1 / 2}\right)$ pour $-1<\mu<0$. Cette fonction sera impaire et discontinue pour $\mu=0$. Si nous lui ajoutons une fonction $v_{2}(\mu)$ égale à $-\left(1-\mu^{2}\right)^{m / 2} u\left((2 \lambda c)^{1 / 2}\right)$ pour $0<\mu<1$ et à $\left(1-\mu^{2}\right)^{m / 2} u\left((2 \lambda c)^{1 / 2}\right)$ pour $-1<\mu<0$, la somme $v(\mu)=v_{1}(\mu)+v_{2}(\mu)$ sera continue dans tout l'intervalle $(-1,+1)$ et de plus, comme $(d / d \mu) \cdot u\left(\left(2 \lambda c\left(1-\mu^{2}\right)\right)^{1 / 2}\right)=0$ pour $\mu=0$, la dérivée de $v(\mu)$ sera également continue dans l'intervalle $(-1,+1)$.

Définissons en suite une nouvelle fonction $V(\mu)=C_{1} v(\mu)$, où $C_{1}$ est un constante telle que

$$
\begin{aligned}
\int_{-1}^{1} V^{2}(\mu) d \mu & =C_{1}^{2} \int_{-1}^{1} v^{2}(\mu) d \mu \\
& =\frac{2}{2 n_{1}+1} \cdot \frac{n_{1}+m !}{n_{1}-m !}
\end{aligned}
$$

et où $n_{1}$ est un entier positif qui sera défini plus loin.

La fonction $V(\mu)$, étant impaire, pourra être développée en série convergente de fonctions $\bar{P} e_{n}^{m}(\mu)$ impaires. Donc

$$
V(\mu)=\sum_{r=m}^{\infty} B_{r} \bar{P} e_{r}^{m}(\mu) .
$$

D'autre part, la fonction $V(\mu)$ vérifiera l'équation différentielle

$$
\frac{d}{d \mu}\left[\left(1-\mu^{2}\right) \frac{d V}{d \mu}\right]+\left(b+\lambda^{2} c^{2} \mu^{2}-\frac{m^{2}}{1-\mu^{2}}\right) V=O\left((\lambda c)^{-k}\right)
$$

car, par suite de la présence du facteur $e^{-\lambda_{0}\left(1-\mu^{2}\right) / 2}$ dans l'expression de $u(\mu)$, on aura, pour $\lambda c$ très grand

$$
u\left((2 \lambda c)^{1 / 2}\right)=O\left(e^{-\lambda_{e} / 2}\right)
$$


et, par conséquent, la fonction $v_{2}(\mu)$ n'introduira dans le second membre de l'équation différentielle que des termes asymptotiquement négligeables vis-àvis de ceux en $(\lambda c)^{-k}$.

En raisonnant maintenant exactement comme dans le cas des fonctions $\bar{P} e_{n}^{m}(\mu)$ paires, nous pourrons montrer que

$$
\begin{aligned}
b & =b_{n_{1}}^{m}+O\left((\lambda c)^{-k}\right), \\
V(\mu) & =\bar{P} e_{n_{1}}^{m}(\mu)+O\left((\lambda c)^{-k}\right) .
\end{aligned}
$$

Si l'on remarque que, comme nous venons de le dire, les termes provenant de la fonction $v_{2}(\mu)$ sont asymptotiquement négligeables, on en déduira que, dans les égalités qui précèdent, on pourra prendre pour $V(\mu)$ simplement la fonction $C_{1} v_{1}(\mu)$, où $C_{1}$ sera défini par

$$
C_{1}^{2} \int_{-1}^{1} v_{1}^{2}(\mu) d \mu=\frac{2}{2 n_{1}+1} \cdot \frac{n_{1}+m !}{n_{1}-m !} .
$$

Ceci nous montre enfin que, entre 0 et $1, V(\mu)$ ne diffère que par un facteur constant de $U(\mu)$ et, entre -1 et 0 , ne diffère que par le même facteur constant, ne $-U(\mu)$.

Ils nous reste plus maintenant qu'à rechercher la relation entre $p$ et $n_{1}$. Remarquons pour cela que, pour $\lambda c$ très grand, $V(\mu)$ différera d'aussi peu qu'on veut, entre 0 et +1 , de

$$
C_{1}\left(1-\mu^{2}\right)^{m / 2} e^{-\lambda c\left(1-\mu^{2}\right) / 2} L_{p}^{(m)}\left[\lambda c\left(1-\mu^{2}\right)\right]
$$

et d'aussi peu qu'on veut de cette même fonction changée de signe pour $\mu$ compris entre -1 et 0 . Or la fonction $L_{p}^{(m)}(z)$, pour $z$ positif, possède exactement $p$ zéros. La fonction $V(\mu)$ possèdera donc en tout, en comptant le zéro $\mu=0,2 p+1$ zéros. D'autre part, la fonction impaire $\bar{P} e_{n_{1}}^{m}(\mu)$ possède $n_{1}-m$ zéros différents de $\mu= \pm 1$. Par conséquent donc

$$
n_{1}-m=2 p+1 \text { ou } n_{1}=2 p+m+1 \text {. }
$$

De tout ceci il résulte que

$$
\begin{aligned}
V(\mu) & =\bar{P} e_{2 p+m+1}^{m}(\mu)+O\left((\lambda c)^{-k}\right), \\
b & =b_{2 p+m+1}^{m}+O\left((\lambda c)^{-k}\right) .
\end{aligned}
$$

La fonction $u\left(\left(2 \lambda c\left(1-\mu^{2}\right)\right)^{1 / 2}\right)$ représente donc, à un facteur constant près, puisque les constantes de normalisation contiennent le nombre $n$, asymptotiquement, pour $0 \leqq \mu \leqq 1$, simultanément les fonctions

$$
\bar{P} e_{2 p+m}^{m}(\mu) \text { et } \bar{P} e_{2 p+m-1}^{m}(\mu) .
$$

Pour $-1 \leqq \mu \leqq 0$, cette même fonction, avec les mêmes facteurs constants, représente asymptotiquement simultanément les deux fonctions 


$$
\bar{P} e_{2 p+m}^{m}(\mu) \text { et }-\bar{P} e_{2 p+m+1}^{m}(\mu) .
$$

De même, la quantité $b$ représente asymptotiquement simultanément les deux constantes de séparation $b_{2 p+m}^{m}$ et $b_{2 p+m+1}^{m}$. Ceci peut se vérifier immédiatement sur la Fig. 1, où nous avons représenté la variation des constantes $a_{n}^{m}$ et $b_{n}^{m}$ (pour $m=0$ ) en fonction de la quantité $(\lambda c)^{2}$. La partie de droite, correspondant à $(\lambda c)^{2}$ positif, se rapporte aux fonctions $P e_{n}^{m}$ relatives à l'ellipsoide allongé, et la partie de gauche, correspondant à $(\lambda c)^{2}$ négatif, se rapporte à l'ellipsoide de révolution aplati. La partie centrale du diagramme a été construite en utilisant les tables de Stratton, Morse, Chu et Hutner, et les parties extrêmes en utilisant les formules asymptotiques obtenues cidessus.

Ce diagramme montre bien comment les deux théories des fonctions d'ondes sphéroidales relatives à l'ellipsoide allongé et à l'ellipsoide aplati n'en font en réalité qu'une seule. Mais il montre aussi, combien, pour $(\lambda c)^{2}$ très grand, sont essentiellement différents les comportements des fonctions des deux types. Nous reviendrons plus loin sur ce point.

Nous allons maintenant donner les valeurs explicites de $u(\alpha)$ et de $b$.

On trouve que

$$
u(\alpha)=\alpha^{m} e^{-\alpha^{2} / 4}\left(w_{0}+w_{1}+w_{2}+\cdots\right)
$$

avec

$$
\begin{aligned}
w_{0}= & L_{p}^{(m)}\left(\frac{\alpha^{2}}{2}\right) \\
w_{1}= & \frac{1}{2 \lambda c}\left[-\frac{1}{4} C_{1, p} L_{p+2}^{(m)}-\frac{1}{2} C_{2, p} L_{p+1}^{(m)}+\frac{1}{2} C_{4, p} L_{p-1}^{(m)}+\frac{1}{4} C_{5, p} L_{p-2}^{(m)}\right] \\
w_{2}= & \frac{1}{(2 \lambda c)^{2}}\left[\frac{1}{32} C_{1, p} C_{1, p+2} L_{p+4}^{(m)}+\frac{1}{24}\left(C_{1, p} C_{2, p+2}+2 C_{1, p} C_{2, p}\right) L_{p+3}^{(m)}\right. \\
& +\frac{1}{16}\left(C_{1, p} C_{3, p+2}+2 C_{2, p} C_{2, p+1}-C_{1, p} C_{3, p}\right) L_{p+2}^{(m)} \\
& +\frac{1}{8}\left(C_{1, p} C_{4, p+2}+2 C_{2, p} C_{3, p+1}-2 C_{4, p} C_{1, p-1}-2 C_{2, p} C_{3, p}\right) L_{p+1}^{(m)} \\
& +\frac{1}{8}\left(-2 C_{2, p} C_{5, p+1}+2 C_{4, p} C_{3, p-1}+C_{5, p} C_{2, p-2}-2 C_{4, p} C_{3, p}\right) L_{p-1}^{(m)} \\
& +\frac{1}{16}\left(2 C_{4, p} C_{4, p-1}+C_{3, p-2} C_{5, p}-C_{5, p} C_{3, p}\right) L_{p-2}^{(m)} \\
& \left.+\frac{1}{24}\left(2 C_{4, p} C_{5, p-1}+C_{4, p-2} C_{5, p}\right) L_{p-3}^{(m)}+\frac{1}{32} C_{5, p} C_{5, p-2} L_{p-4}^{(m)}\right]
\end{aligned}
$$


Ensuite

$$
b=-(\lambda c)^{2}+2(m+2 p+1) \lambda c+\gamma_{0}+\frac{\gamma_{1}}{2 \lambda c}+\frac{\gamma_{2}}{(2 \lambda c)^{2}}+\cdots
$$

avec

$$
\begin{aligned}
\gamma_{0}= & C_{3, p}, \\
\gamma_{1}= & -\frac{1}{4} C_{1, p} C_{5, p+2}-\frac{1}{2} C_{2, p} C_{4, p+1}+\frac{1}{2} C_{4, p} C_{2, p-1}+\frac{1}{4} C_{5, p} C_{1, p-2}, \\
\gamma_{2}= & \frac{1}{16} C_{5, p+2}\left(C_{1, p} C_{3, p+2}+2 C_{2, p} C_{2, p+1}-C_{1, p} C_{3, p}\right) \\
& +\frac{1}{8} C_{4, p+1}\left(C_{1, p} C_{4, p+2}+2 C_{2, p} C_{3, p+1}-2 C_{4, p} C_{1, p-1}-2 C_{2, p} C_{3, p}\right) \\
& +\frac{1}{8} C_{2, p-1}\left(-2 C_{2, p} C_{5, p+1}+2 C_{4, p} C_{3, p-1}+C_{5, p} C_{2, p-2}-2 C_{4, p} C_{3, p}\right) \\
& +\frac{1}{16} C_{1, p-2}\left(2 C_{4, p} C_{4, p-1}+C_{3, p-2} C_{5, p}-C_{5, p} C_{3, p}\right) .
\end{aligned}
$$

Pour faciliter l'emploi de ces formules, nous donnons ci-après un tableau des valeurs des coefficients $C$.

\begin{tabular}{l|l|l|l|l}
$C_{1,0}=2$ & $C_{2,0}=-1$ & $C_{3,0}=-(1+m)$ & $C_{4,0}=m$ & $C_{5,0}=m(m-1)$ \\
$C_{1,1}=6$ & $C_{2,1}=-2$ & $C_{3,1}=-(5+3 m)$ & $C_{4,1}=m+1$ & $C_{5,1}=m(m+1)$ \\
$C_{1,2}=12$ & $C_{2,2}=-3$ & $C_{3,2}=-(13+5 m)$ & $C_{4,2}=m+2$ & $C_{5,2}=(m+1)(m+2)$ \\
$C_{1,3}=20$ & $C_{2,3}=-4$ & $C_{3,3}=-(25+7 m)$ & $C_{4,3}=m+3$ & $C_{5,3}=(m+2)(m+3)$ \\
$C_{1,4}=30$ & $C_{2,4}=-5$ & $C_{3,4}=-(41+9 m)$ & $C_{4,4}=m+4$ & $C_{5,4}=(m+3)(m+4)$ \\
$C_{1,5}=42$ & $C_{2,5}=-6$ & $C_{3,5}=-(61+11 m)$ & $C_{4,5}=m+5$ & $C_{b, 5}=(m+4)(m+5)$ \\
$C_{1,6}=56$ & $C_{2,6}=-7$ & $C_{3,6}=-(85+13 m)$ & $C_{4,6}=m+6$ & $C_{b, 6}=(m+5)(m+6)$
\end{tabular}

En effectuant les calculs, on trouve en particulier, et en ne tenant pas compte des termes en $(\lambda c)^{-3}$,

$p=0$

$$
\begin{aligned}
u= & \alpha^{m} \mathrm{e}^{-\alpha^{2} / 4}\left[L_{0}^{(m)}+\frac{1}{2 \lambda c}\left(-\frac{1}{2} L_{2}^{(m)}+\frac{1}{2} L_{1}^{(m)}\right)\right. \\
& +\frac{1}{(2 \lambda c)^{2}}\left(\frac{3}{4} L_{4}^{(m)}-\frac{5}{12} L_{3}^{(m)}-\frac{2 m+5}{4} L_{2}^{(m)}+\frac{3(m+2)}{4} L_{1}^{(m)}\right) \\
b= & -(\lambda c)^{2}+2(m+1) \lambda c-(m+1) \\
& -\frac{(m+1)^{2}}{2} \cdot \frac{1}{2 \lambda c}-\frac{(m+1)^{2}(m+2)}{2} \cdot \frac{1}{(2 \lambda c)^{2}}
\end{aligned}
$$


$p]=1$

$$
\begin{aligned}
u= & \alpha^{m} e^{-\alpha^{2} / 4}\left[L_{1}^{m}+\frac{1}{2 \lambda c}\left(-\frac{3}{2} L_{3}^{m}+L_{2}^{m}+\frac{m+1}{2} L_{0}^{m}\right)\right. \\
& +\frac{1}{(2 \lambda c)^{2}}\left(\frac{15}{4} L_{5}^{m}-2 L_{4}^{m}-\frac{3(2 m+9)}{4} L_{3}^{m}+\frac{5 m+23}{4} L_{2}^{m}\right. \\
& \left.\left.+(m+1)(m+2) L_{0}^{m}\right)\right], \\
b= & -(\lambda c)^{2}+2(m+3) \lambda c-(3 m+5) \\
& -\frac{(m+3)(3 m+5)}{2} \cdot \frac{1}{2 \lambda c}-\frac{(m+2)\left(3 m^{2}+22 m+31\right)}{2} \cdot \frac{1}{(2 \lambda c)^{2}} .
\end{aligned}
$$

Il nous faut maintenant déterminer la valeur asymptotique de la constante de normalisation. Celle-ci est donnée, dans tous les cas, par l'équation

$$
\begin{aligned}
\frac{C^{2}}{(2 \lambda c)^{m+1}} \int_{0}^{(2 \lambda c)^{1 / 2}} \alpha^{2 m+1} e^{-\alpha^{2} / 2}\left(w_{0}+w_{1}+w_{2}+\cdots\right)^{2}(1- & \left.\frac{\alpha^{2}}{2 \lambda c}\right)^{-1 / 2} d \alpha \\
& =\frac{1}{2 n+1} \cdot \frac{n+m !}{n-m !} .
\end{aligned}
$$

En raisonnant comme dans le cas des fonctions de Mathieu, on démontrera que, pour le calcul de la valeur asymptotique de $C$, on peut remplacer, dans l'intégrale, $\left(1-\alpha^{2} / 2 \lambda c\right)^{-1 / 2}$ par son développement.en série de puissances de $\alpha^{2} / 2 \lambda c$, et qu'on peut ensuite prendre pour limite supérieure de l'intégrale $\infty$ au lieu de $(2 \lambda c)^{1 / 2}$. Dans ces conditions, on trouve, pour la valeur asymptotique de l'intégrale

$$
\begin{array}{r}
\int_{0}^{\infty} \alpha^{2 m+1} e^{-\alpha^{2} / 2}\left(w_{0}+w_{1}+w_{2}+\cdots\right)^{2}\left(1+\frac{\alpha^{2}}{4 \lambda c}+\frac{3 \alpha^{4}}{8(\lambda c)^{2}}+\cdots\right) d \alpha \\
=2^{m \cdot \frac{p+m !}{p !}}\left[c_{0}+\frac{c_{1}}{2 \lambda c}+\frac{c_{2}}{(2 \lambda c)^{2}}+\cdots\right]
\end{array}
$$

avec

$$
\begin{aligned}
c_{0}= & 1, \quad c_{1}=m+2 p+1, \\
c_{2}= & 6\left[(p+1)(m+p+1)+(m+2 p+1)^{2}+p(p+m)\right] \\
& -[p(p+m)+(p+1)(p+m+1)] \\
& +\frac{1}{4}\left[\frac{(p+1)(m+p+1)(p+2)(m+p+2)}{4}\right. \\
& +(p+1)(m+p+1)+p(m+p)] .
\end{aligned}
$$

Il en résulte que $C$ sera donné par 


$$
C=\left[2 \cdot \frac{(\lambda c)^{m+1}}{2 n+1} \cdot \frac{n+m ! p !}{n-m ! p+m !}\right]^{1 / 2} /\left[c_{0}+\frac{c_{1}}{2 \lambda c}+\frac{c_{2}}{(2 \lambda c)^{2}}+\cdots\right]^{1 / 2}
$$

Rappelons que, dans ces formules, $p=(n-m) / 2$ ou $(n-m-1) / 2$, suivant $n-m$ est pair ou impair. On trouve ainsi en particulier

$n=0, m=0, p=0$

$$
C=(2 \lambda c)^{1 / 2} /\left(1+\frac{1}{2 \lambda c}+\frac{23}{2} \cdot \frac{1}{(2 \lambda c)^{2}}\right)^{1 / 2} .
$$

$n=1, m=0, p=0$

$$
C=\left(\frac{2 \lambda c}{3}\right)^{1 / 2} /\left(1+\frac{1}{2 \lambda c}+\frac{23}{2} \cdot \frac{1}{(2 \lambda c)^{2}}\right)^{1 / 2} .
$$

$n=1, m=1, p=0$

$$
C=\frac{2 \lambda c}{3^{1 / 2}} /\left(1+\frac{2}{2 \lambda c}+\frac{141}{4} \cdot \frac{1}{(2 \lambda c)^{2}}\right)^{1 / 2} .
$$

$n=2, m=0, p=1$

$$
C=\left(\frac{2 \lambda c}{5}\right)^{1 / 2} /\left(1+\frac{3}{2 \lambda c}+\frac{165}{2} \cdot \frac{1}{(2 \lambda c)^{2}}\right)^{1 / 2} .
$$

Comme nous l'avons fait plus haut pour les fonctions associées à l'ellipsoide allongé, nous allons maintenant comparer les valeurs que fournissent les formules asymptotiques qui précèdent avec les valeurs exactes des fonctions et des constantes de séparation.

En ce qui concerne les fonctions sphéroidales, les seules tables existantes sont à nouveau celles publiées par Stratton, Morse, Chu et Hutner, lesquelles ne donnent que les valeurs correspondant à des valeurs de $\lambda c \leqq 5$. Mais, en ce qui concerne les constantes de séparation, une série de valeurs de celles-ci, en particulier pour $\lambda c=8$, ont été données par Hylleraas.

Ajoutons, pour être complet, que des formules asymptotiques pour les constantes de séparation, équivalentes aux nôtres, ont été publiées par Hylleraas et par Baber et Hasse.

Le tableau qui suit permet de comparer les valeurs exactes de $b_{n}^{m}$ pour $\lambda c=5$ et $\lambda c=8$, avec les valeurs approchées fournies par nos formules.

Comme on s'en rend immédiatement compte, les formules asymptotiques donnent, pour les constantes de séparation, des valeurs remarquablement exactes dès que $\lambda c$ est assez grand pour que $b_{n}^{m}$ et $b_{n+1}^{m}$ ne soient que très peu différents. D'autre part, l'erreur commise croît rapidement, pour $\lambda c$ donné, au fur et à mesure que $m$ et $p$ deviennent plus grands. Ceci n'a rien d'étonnant si on se reporte à la Fig. 1 , puisque, pour $\lambda c=0, b_{n}^{m}$, et $b_{n+1}^{m}$ valent respective- 
ment $n(n+1)$ et $(n+1)(n+2)$ c'est à dire des nombres dont la différence augmente rapidement avec $n$.

Passons maintenant aux fonctions $\bar{P} e_{n}^{m}(\mu)$ elles mêmes. Dans les tables calculées par Stratton et al, ces fonctions sont normalisées par la condition que, pour $\mu=0$, la fonction $\bar{P} e_{n}^{m}(\mu)$ a la même valeur que $P_{n}^{m}(\mu)$, et il a été tenu compte de cette différence dans les valeurs numériques qui suivent.

Pour $m \neq 0$, les fonctions $\bar{P} e_{n}^{m}(\mu)$ sont évidemment nulles pour $\mu=1$, mais la limite du rapport $\bar{P} e_{n}^{m}(\mu) /\left(1-\mu^{2}\right)^{m / 2}$ est différente de zéro. Nous avons calculé cette limite pour $\lambda c=5$ et différentes valeurs de $m$ et $n$, et l'avons comparée aux valeurs exactes. Voici les résultats obtenus.

On a tout d'abord, pour les valeurs générales de $\lambda c$,

\begin{tabular}{|c|c|c|c|c|c|c|}
\hline \multirow[b]{2}{*}{$m$} & \multirow[b]{2}{*}{$n$} & \multirow[b]{2}{*}{$p$} & \multicolumn{2}{|c|}{$c=5$} & \multicolumn{2}{|c|}{$c=8$} \\
\hline & & & $b$ exact & $\begin{array}{l}b \text { approxi- } \\
\text { matif }\end{array}$ & $b$ exact & $\begin{array}{l}b \text { approxi- } \\
\text { matif }\end{array}$ \\
\hline 0 & 0 & 0 & $-16,0790$ & & $-49,0362$ & \\
\hline 0 & 1 & 0 & $-16,0504$ & $-16,0600$ & $-49,0360$ & $-49,0356$ \\
\hline 0 & 2 & 1 & $-2,4486$ & & $-21,7226$ & \\
\hline 0 & 3 & 1 & 0,0609 & $-1,0600$ & $-21,6178$ & $-21,5898$ \\
\hline 1 & 1 & 0 & $-7,4934$ & & $-34,1602$ & \\
\hline 1 & 2 & 0 & $-7,1278$ & $-7,2600$ & $-34,1551$ & $-34,1484$ \\
\hline 1 & 3 & 1 & 2,7504 & & $-10,1067$ & \\
\hline 1 & 4 & 1 & $\ldots$ & 4,5600 & $-9,2422$ & $-9,3281$ \\
\hline 2 & 2 & 0 & 0,4289 & & $-19,4238$ & \\
\hline 2 & 3 & 0 & 2,1098 & 1,3700 & $-19,3656$ & $-19,3516$ \\
\hline 3 & 3 & 0 & 8,3077 & & $-4,9915$ & \\
\hline 3 & 4 & 0 & $\cdots$ & 9,8000 & $-4,6116$ & $-4,6562$ \\
\hline
\end{tabular}

Ensuite

$$
\bar{P} e_{0}(1) \sim(2 \lambda c)^{1 / 2}\left(1+\frac{7}{12} \cdot \frac{1}{(2 \lambda c)^{2}}\right) /\left(1+\frac{1}{2 \lambda c}+\frac{23}{2} \cdot \frac{1}{(2 \lambda c)^{2}}\right)^{1 / 2},
$$




$$
\begin{gathered}
\bar{P} e_{1}(1) \sim\left(\frac{2 \lambda c}{3}\right)^{1 / 2}\left(1+\frac{7}{12} \cdot \frac{1}{(2 \lambda c)^{2}}\right) /\left(1+\frac{1}{2 \lambda c}+\frac{23}{2} \cdot \frac{1}{(2 \lambda c)^{2}}\right)^{1 / 2}, \\
\bar{P} e_{2}(1) \sim\left(\frac{2 \lambda c}{5}\right)^{1 / 2}\left(1+\frac{11}{4} \cdot \frac{1}{(2 \lambda c)^{2}}\right) /\left(1+\frac{3}{2 \lambda c}+\frac{165}{2} \cdot \frac{1}{(2 \lambda c)^{2}}\right)^{1 / 2}, \\
\lim _{\mu \rightarrow 1} \frac{\bar{P} e_{1}^{1}(\mu)}{\left(1-\mu^{2}\right)^{1 / 2}} \\
\sim \frac{2 \lambda c}{3^{1 / 2}}\left(1-\frac{1}{2} \cdot \frac{1}{2 \lambda c}+\frac{4}{3} \cdot \frac{1}{(2 \lambda c)^{2}}\right) /\left(1+\frac{2}{2 \lambda c}+\frac{141}{4} \cdot \frac{1}{(2 \lambda c)^{2}}\right)^{1 / 2} .
\end{gathered}
$$

En faisant dans ces formules $\lambda c=5$, on trouve

\begin{tabular}{|c|c|c|}
\hline & approximatif & exact \\
\hline $\bar{P} e_{0}(1)$ & 2,8856 & 2,8646 \\
$\bar{P} e_{1}(1)$ & 1,6600 & 1,7230 \\
$\bar{P} e_{2}(1)$ & 0,9968 & 0,9683 \\
$\lim _{\mu \rightarrow 1} \frac{\bar{P} e_{1}^{1}(\mu)}{\left(1-\mu^{2}\right)^{1 / 2}}$ & 4,4689 & 4,6055 \\
\hline
\end{tabular}

Encore une fois, l'approximation est bonne pour $n=0$, mais devient rapidement plus mauvaise dès que $m$ ou $n$ augmentent. Il serait intéressant de pouvoir faire la comparaison pour des valeurs plus élevées de $\lambda c$ car, comme le montre le tableau relatif aux constantes de séparation, l'exactitude augmente très rapidement avec $\lambda c$.

Voyons, pour finir, quels sont les renseignement que nous donnent les formules asymptotiques sur le comportement des fonctions $\bar{P} e_{n}^{m}(\mu)$, pour $-1 \leqq \mu \leqq 1$, réel, et pour $\lambda c$ croissant indéfiniment.

La présence du facteur $e^{\lambda c /\left(1-\mu^{2}\right) 2}$ nous montre tout d'abord que, pour $\mu$ fixe et $\neq \pm 1$, la fonction $\bar{P} e_{n}^{m}(\mu)$ tend uniformément vers zéro lorsque $\lambda c$ augmente indéfiniment.

D'autre part, tous les zéros non nuls de la fonction se rapprocheront de plus en plus des points $\mu= \pm 1$, ainsi, bien entendu, que les maxima et minima qui les séparent.

Pour $\mu= \pm 1, \bar{P} e_{n}^{m}(\mu)$ est nul, sauf si $m=0$, mais le rapport

$$
\frac{\bar{P} e_{n}^{m}(\mu)}{\left(1-\mu^{2}\right)^{m / 2}}
$$

tend vers une limite finie lorsque $\mu$ tend vers \pm 1 . Lorsque $\lambda c$ croît indéfiniment, cette limite devient également infinie, mais de telle manière que 


$$
\lim _{\lambda c \rightarrow \infty} \frac{1}{(\lambda c)^{(m+1) / 2}}\left[\lim _{\mu \rightarrow 1} \frac{\bar{P} e_{n}^{m}(\mu)}{\left(1-\mu^{2}\right)^{m / 2}}\right]=\left[\frac{2}{2 n+1} \cdot \frac{n+m !}{n-m !} \cdot \frac{p+m !}{p ! m !}\right]^{1 / 2} .
$$

Ce qui précède montre bien la différence fondamentale qui existe entre les comportements asymptotiques des fonctions $P e_{n}^{m}(\mu)$ et $\bar{P} e_{n}^{m}(\mu)$ et qui résulte seulement du remplacement de $(\lambda c)^{2}$ par $-(\lambda c)^{2}$ dans l'équation différentielle qui les définit.

Lorsque $\lambda c$ devient très grand, la fonction $P e_{n}^{m}(\mu)$ tend vers zéro pour toutes les valeurs de $\mu$ différentes de $\mu=0$, tandis que, dans les mêmes conditions, la fonction $\bar{P} e_{n}^{m}(\mu)$ tend vers zéro pour toutes les valeurs de $\mu$ différentes de $\mu= \pm 1$. Les deux fonctions, pour $\lambda c$ très grand, ont en quelque sorte leurs valeurs non nulles concentrées aux environs de $\mu=0$, pour $P e_{n}^{m}(\mu)$, et aux environs de $\mu= \pm 1$, pour $\bar{P} e_{n}^{m}(\mu)$.

Si l'on se reporte aux coordonnées sphéroidales définies dans l'introduction, on voit que, dans le cas de l'ellipsoide allongé, $\mu=\cos \eta=0$ représente le plan équatorial $z=0$, tandis que, pour l'ellipsoide aplati, $\mu=\sin \eta= \pm 1$ représente l'axe de révolution $x=y=0$.

\section{BiBliographie}

S. Goldstein, Mathieu functions, Trans. Cambridge Philos. Soc. vol. 23 (1927) pp. 303-336.

E. L. Ince, The Mathieu equation with numerically large parameters, J. London Math. Soc. vol. 2 (1927) pp. 46-50. pp. $355-433$.

, Tables of the elliptic-cylinder functions, Proc. Roy. Soc. Edinburgh vol. 52 (1932)

N. G. Baber and H. R. Hasse, The two center problem in wave mechanics, Proc. Cambridge Philos. Soc. vol. 31 (1935) pp. 564-581.

E. A. Hylleraas, Equation d'ondes d'un électron dans le champ de force de deux noyaux atomiques. Problème de l'ion moléculaire d'hydrogène, Annales Institut Henri Poincaré vol. 7 (1937) pp. 121-153.

J. A. Stratton, P. M. Morse, L. J. Chu and R. R. Hutner, Elliptic cylinder and spheroidal wave functions, New York, Wiley, 1941.

Union Chimique Belge,

Brussels, Belgium 